\title{
Clustering of the optical-afterglow luminosities of long gamma-ray bursts ${ }^{\star}$
}

\author{
M. Nardini ${ }^{1,2}$, G. Ghisellini ${ }^{1}$, G. Ghirlanda ${ }^{1}$, F. Tavecchio ${ }^{1}$, C. Firmani ${ }^{1,3}$, and D. Lazzati ${ }^{4}$ \\ ${ }^{1}$ Osservatorio Astronomico di Brera, via Bianchi 46, 23807 Merate, Italy \\ e-mail: gabriele@merate.mi .astro.it \\ 2 Univ. di Milano-Bicocca, P.za della Scienza 3, 20126 Milano, Italy \\ 3 Instituto de Astronomía, UNAM, AP 70-264, 04510 México, DF, México \\ 4 JILA, University of Colorado, Boulder, CO 80309-0440, USA
}

Received 22 August 2005 / Accepted 16 January 2006

ABSTRACT

\begin{abstract}
We studied the optical afterglows of the 24 pre-SWIFT gamma-ray bursts (GRBs) with both known spectroscopic redshift and published estimates of the optical extinction in the source frame. We found an unexpected clustering of the optical-afterglow luminosities measured $12 \mathrm{~h}$ (source frame time) after the trigger. For 21 out of 24 bursts, the distribution of the optical luminosities is narrower than the distribution of the X-ray luminosities, and even narrower than the distribution of the ratio between the monochromatic optical luminosities and the total isotropic, emitted prompt energy. Three bursts stand out from the distribution of the other sources, being underluminous by a factor $\sim 15$. We compare this result with another somewhat analogous result concerning the luminosity of the X-ray afterglows studied earlier. We constructed the optical to X-ray spectral energy distribution for all our GRBs. For all but a minority of them, the optical and the X-ray emissions are consistent with being produced by the same radiation process. We discuss our results in the framework of the "standard" external-shock synchrotron model. Finally, we consider the behavior of the first GRBs of known redshifts detected by SWIFT. We find that these SWIFT GRBs entirely confirm our findings.
\end{abstract}

Key words. gamma rays: bursts - radiation mechanisms: non-thermal - X-rays: general

\section{Introduction}

The commonest approach to comparing directly the afterglow emission of different bursts is to compute the light curves in the observer's reference frame, in terms of their fluxes vs. the $o b$ served time $t_{\mathrm{obs}}$. However, when the redshift is known, a more fruitful approach is to compare the light curves of the luminosities of different bursts, using the rest-frame time $t_{\mathrm{RF}}=$ $t_{\mathrm{obs}} /(1+z)$. Although such attempts have already been done (see, e.g. Gendre \& Boër 2005, hereafter GB05; Kumar \& Piran 2000), they mainly concerned the X-ray luminosities of relatively small samples of GRBs, and not the optical luminosities (but see Berger et al. 2005, for a study of the first SWIFT bursts). From these earlier studies (see also Piran et al. 2000) it appeared that the X-ray afterglow luminosities (calculated at the same time in the rest frame) were characterized by a smaller dispersion than the dispersion of the total energies radiated during the prompt emission (but see Berger et al. 2003, for a different conclusion). In addition, Boër \& Gendre (2000) and GB05 found that the X-ray afterglow luminosities showed a tendency to cluster in two groups (different by a factor $\sim 30$ in luminosity) with a small dispersion within each group. These authors also tried to draw conclusions from the optical luminosity, but were not successful because the absorption was largely unknown at that time, and because of too small a sample.

These earlier results prompted us to study the behavior of the optical afterglow luminosities. One of the main initial motivations of our study was the possibility that what seems to be

^ Appendix A is only available in electronic form at http://www. edpsciences.org a "dichotomy" in the X-ray afterglow luminosity could also be present in the optical, therefore helping to understand the problem of the so-called "dark" bursts, those with a detected X-ray afterglow but no optical detection. De Pasquale et al. (2003), when comparing GRBs (all with detected X-ray afterglow) with and without optical detection, found that "dark" GRBs tend to be fainter in the X-ray, by a factor $\sim 5$ in flux at the same observed time. We, however, expect a greater dispersion of the optical luminosity (at a given rest-frame time) than the corresponding dispersion of the X-ray luminosities. This is because electrons emitting X-rays by the synchrotron process probably cool on a dynamical timescale (also several hours after trigger), and this implies (in the standard synchrotron fireball model) that the emitted X-ray emission is insensitive to the density of the external medium producing the external shocks. On the contrary, it is likely that electrons emitting in the optical do not cool in a dynamical timescale (about a day after the prompt phase), so the optical emission does depend on the density of the circumburst material. If the dispersion introduced by this effect is not too large, some sort of "dichotomy" could survive and then flag the existence of two families of GBRs with two different average afterglow luminosities. Dark GRBs could then be thought to belong to the underluminous family, therefore be more difficult to detect. This is all the more so in the optical, if some extinction in the host galaxy is present.

The results presented in the following are instead quite puzzling, since - contrary to the simple expectations mentioned above - the optical afterglow luminosities show a degree of clustering that is tighter than the one shown by the X-ray afterglow luminosities. We indeed find an indication (albeit still weak, due 
to small statistics) of a dichotomy in the optical luminosity distributions. But, more intriguingly, we find an unexpected optical luminosity clustering of the large majority of the bursts analyzed by us (21 out of 24). In order to understand it, we have constructed, for all GRBs of our sample, the optical to X-rays spectral energy distribution (SED) at a given time, assembling information contained in the optical multiband photometry and in the X-ray continuum flux and spectrum. This allows to see if both the optical and the X-ray fluxes are consistent with being produced by a single electron population by the synchrotron process, or if there is some indication of X-ray fluxes being produced by an additional component (i.e. a possibly emergent inverse Compton flux in the X-ray band), and therefore responsible for the larger dispersion of X-ray luminosities with respect to the optical ones. As we will show, this is not the case for most of the sources.

We also consider in Sect. 5 the bursts detected by SWIFT and for which the redshift is known. All of them but GRB 050401, GRB 050525, and GRB 050730 lack information about the optical absorption in their hosts. With this caveat, we calculate their optical luminosities and find that they are consistent with the clustering properties of the other bursts. Instead, we find that, on average, they are more powerful in X-rays with respect to the pre-SWIFT bursts so they broaden the X-ray luminosity distribution. We finally discuss these results in the framework of the standard fireball external shocks synchrotron scenario, and the possible implications for dark bursts.

Throughout this paper, we adopt a cosmology with $\Omega_{\mathrm{M}}=0.3$ and $\Omega_{\Lambda}=h_{0}=0.7$.

\section{The sample}

To compare the rest frame optical luminosities of different GRBs, we applied all the relevant cosmological and extinction corrections to the GRB light curves. In particular, one of our selection criteria is that the absorption $A_{V}^{\text {host }}$ in the host galaxy is already known from the literature.

We collected from the literature all GRBs of known spectroscopic redshift $z$, detected optical afterglow, known optical spectral index, and known optical extinction in the host rest frame $A_{V}^{\text {host }}$. The total number of GRBs with measured redshifts is more than 50 (as of July 31st, 2005) and 24 of those fulfilling our selection criteria. They are listed in Table 1. This list includes 13 out of the 17 GRBs present in the list of GB05. The 4 missing GRBs are: GRB 970228 (for which there is no estimate of $A_{V}^{\text {host }}$ ), GRB 000210 and GRB 000214 (with no detected optical afterglow), and GRB 980425 (an anomalous GRB associated with the 1998bw supernova).

In Table 1 we report, for every GRB in our sample, the redshift, the optical spectral index $\beta_{\mathrm{o}}$, the Galactic absorption $A_{V}^{\mathrm{Gal}}$ (taken from Schlegel 1998 maps except for GRB 011121 for which we also report the values quoted in the corresponding references), the host rest frame absorption $A_{V}^{\text {host }}$, the absorption $A_{v_{R}(1+z)}^{\text {host }}$ at the rest-frame frequency $v_{R}(1+z)$, the extinction and k-corrected monochromatic luminosity $L_{v_{R}}$ (at the source frame frequency corresponding to the $R$ band) and the references of the optical spectral index and extinction value.

Figure 1 shows the behavior of the observed $R$-band fluxes as a function of the observed time $t_{\mathrm{obs}}$ for all bursts listed in Table 1 . In this figure the fluxes are corrected for the Galactic extinction only. In Fig. 2 we show the distribution of the observed fluxes at the same observed time ( $12 \mathrm{~h}$ after trigger). Fitting the distribution of the observed optical fluxes with a Gaussian gives a (logarithmic) dispersion of $\sigma=0.48$ (see Table 2). Note that the Gaussian fit is poor, and the real distribution could have an even larger dispersion.

The monochromatic optical luminosities can be calculated from the observed monochromatic flux $F(v, t)$ by applying the cosmological spectral and time corrections, as

$$
L(v, t)=\frac{4 \pi d_{\mathrm{L}}^{2} F(v, t)}{(1+z)^{1-\beta+\alpha}}
$$

where $d_{\mathrm{L}}$ is the luminosity distance, and we assumed $F(v, t) \propto$ $v^{-\beta} t^{-\alpha}$. Due to the much denser sampling in the Cousin $R$ band, we assumed this band as the optical reference band for all the light curves ${ }^{1}$. We then calculated all monochromatic optical luminosities at the rest frame wavelength of $6400 \AA$ (corresponding to the Cousin $R$ filter $)^{2}$.

All luminosities are given at the same rest-frame time after trigger, which we chose to be $12 \mathrm{~h}$. This choice satisfies the following requirements: i) the data sampling is maximized; ii) for the large majority of bursts, the jet break has not yet occurred; iii) it allows an easy comparison with the X-ray luminosities calculated by GB05, calculated at the same time. For densely sampled optical light curves, we have directly taken the flux measured at $t_{\mathrm{obs}}=12(1+z) \mathrm{h}$. When this flux was not available, we interpolated between data before and after this time. There are 2 cases (GRB 020813 and GRB 030329) in which a break in the light curve (very likely a jet break) occurs before $12 \mathrm{~h}$. In these cases we have extrapolated the flux from data before the break time.

The observed flux $F\left(v_{\mathrm{R}}, t\right)$ was corrected for both galactic and rest-frame extinction. We calculated the $A_{\lambda}$ values for the extinction in the burst's host galaxies by assuming the extinction curve of the Milky Way (Pei 1992), evaluated at the wavelength $\lambda=6400 /(1+z) \AA$ (unless specifically stated otherwise in the original reference). There are cases in which different authors find slightly different values for $A_{V}^{\text {host }}$ and for $\beta_{0}$, i.e. the dereddened value of the optical spectral index. There is some degeneracy between these two quantities when the available data are poorly sampled and affected by relatively large uncertainties. In the large majority of cases, the method used to find the intrinsic extinction is to assume that the spectrum is a power law, and the fit returns the best values of the spectral index and $A_{V}^{\text {host }}$. The two quantities are, however, somewhat correlated, since increasing $A_{V}^{\text {host }}$ gives a flatter $\beta$. In addition, different results can be obtained by using different extinction curves. Therefore, for completeness, we list the different values of $A_{V}^{\text {host }}$ and $\beta_{\mathrm{o}}$ found by different authors in Table 1 , and the corresponding value of the optical luminosity. The first line of every multiple entry in Table 1 corresponds to what we used for the histograms for Fig. 4 and for the following analysis. However, one can see that the different values of the extinction and spectral indices do not change the derived luminosities by a large amount. Indeed, the width of the optical luminosity distribution is not affected by these uncertainties.

Figure 3 shows the distribution of the extinction values $A_{V}^{\text {host }}$ in the host. Despite nearly half of the bursts have zero or almostzero host absorption, the extinction correction is crucial to obtain the strong clustering of the optical luminosities shown in

\footnotetext{
1 We appropriately convert Johnson $R$ magnitudes $(\lambda=6800 \AA)$ into Cousin magnitudes when data in the former filter are given.

2 Note that Eq. (1) is equivalent to Eq. (8) of Lamb \& Reichart (2000), who used the comoving distance instead of the luminosity distance used here, and defined decay and spectral index with opposite signs.
} 
Table 1. Optical properties of GRBs in our sample. References are given for $\beta_{0}, A_{R}^{\mathrm{Gal}}, A_{V}^{\text {host }}$ and $A_{R(1+z)}^{\text {host }}$.

\begin{tabular}{|c|c|c|c|c|c|c|c|c|}
\hline GRB & $z$ & $\operatorname{ref} z$ & $\beta$ & $A_{R}^{\mathrm{Gal}}$ & $A_{V}^{\text {host }}$ & $A_{R(1+z)}^{\text {host }}$ & $\log L_{v_{R}}^{12 \mathrm{~h}}$ & Ref. \\
\hline 970508 & 0.835 & me97 & 1 & 0.13 & 0 & 0 & 30.42 & ga98 \\
\hline 971214 & 3.418 & ku98 & $1.03 \pm 0.18$ & 0.04 & $0.38 \pm 0.08$ & 0.99 & 30.39 & wi99 \\
\hline 980613 & 1.0964 & dj99 & $0.59 \pm 0.03$ & 0.23 & & 0.45 & 29.31 & hj02 \\
\hline \multirow[t]{2}{*}{980703} & 0.966 & $\operatorname{dj} 98$ & $1.01 \pm 0.01$ & 0.15 & $1.51 \pm 0.11$ & 2.50 & 30.82 & vr99 \\
\hline & & & 0.78 & 0.15 & $0.90 \pm 0.20$ & 1.48 & 30.34 & bl98 \\
\hline 990123 & 1.6 & ke99 & $0.750 \pm 0.068$ & 0.04 & 0 & 0 & 30.62 & ho00 \\
\hline 990510 & 1.619 & vr99b & $\begin{array}{l}0.49 \pm 0.1 \\
0.55 \pm 0.1\end{array}$ & $\begin{array}{l}0.54 \\
0.48\end{array}$ & 0 & 0 & $\begin{array}{l}30.73 \\
30.75\end{array}$ & $\begin{array}{l}\text { ho00 } \\
\text { be99 }\end{array}$ \\
\hline 991216 & 1.02 & vr99c & $0.58 \pm 0.08$ & 1.67 & 0 & 0 & 30.89 & ga00 \\
\hline $000301 \mathrm{c}$ & 2.0670 & $\mathrm{fe} 00$ & $0.70 \pm 0.09$ & 0.13 & $0.09 \pm 0.04$ & 0.26 & 30.99 & $\mathrm{je} 01$ \\
\hline 000418 & 1.1181 & bl00 & 0.81 & 0.08 & $0.96 \pm 0.20$ & 1.69 & 30.71 & $\mathrm{kl} 00$ \\
\hline 000911 & 1.06 & pr02 & 1.3 & 0.30 & 0.39 & 0.69 & 30.66 & $\mathrm{ma} 05$ \\
\hline 000926 & 2.0375 & fy 00 & $1.0 \pm 0.2$ & 0.06 & $0.18 \pm 0.06$ & 0.53 & 31.06 & fy01 \\
\hline \multirow[t]{3}{*}{010222} & 1.477 & jh01b & $1.07 \pm 0.09$ & 0.06 & 0 & 0 & 30.52 & st01 \\
\hline & & & $0.89 \pm 0.03$ & & 0 & 0 & 30.45 & jh01 \\
\hline & & & 0.5 & & 0.19 & 0.42 & 30.46 & le01 \\
\hline 010921 & 0.45 & dj01 & $p=3.03$ & 0.396 & $1.16 \pm 0.07$ & 1.43 & 30.77 & pr02a \\
\hline \multirow[t]{3}{*}{011121} & 0.36 & in01 & $0.62 \pm 0.05$ & 1.32 & 0 & 0 & 29.65 & gr03 \\
\hline & & & $0.76 \pm 0.15$ & 0.97 & 0 & 0 & 29.58 & pr02b \\
\hline & & & $0.66 \pm 0.13$ & 1.12 & 0 & 0 & 29.53 & ga03 \\
\hline \multirow[t]{2}{*}{011211} & 2.14 & g101 & $0.56 \pm 0.19$ & 0.11 & $0.08 \pm 0.08$ & 0.23 & 30.36 & ja03 \\
\hline & & & $0.61 \pm 0.15$ & & 0.06 & 0.177 & 30.29 & ho02 \\
\hline \multirow{3}{*}{020124} & 3.198 & at05 & $1.32 \pm 0.25$ & 0.14 & 0 & 0 & 30.22 & hj03 \\
\hline & & & $0.31 \pm 0.43$ & & $2.66 \pm 0.16$ & 0.73 & 29.81 & hj03 \\
\hline & & & $0.91 \pm 0.14$ & & 0 & 0 & 30.00 & hj03 \\
\hline 020405 & 0.69 & $\mathrm{ma} 02$ & 1.45 & 0.15 & 0 & 0 & 30.44 & be 03 \\
\hline 020813 & 1.25 & fi02 & $0.85 \pm 0.07$ & 0.30 & $0.12 \pm 004$ & 0.226 & 30.57 & $\mathrm{co} 03$ \\
\hline 021004 & 2.3351 & gi02 & $0.60 \pm 0.02$ & 0.16 & 0.15 & 0.39 & 31.17 & pa03 \\
\hline \multirow{2}{*}{021211} & 1.004 & vr03 & $0.55 \pm 0.10$ & 0.07 & & 0.48 & 29.41 & fo03 \\
\hline & & & $0.69 \pm 0.14$ & & 0 & 0 & 29.27 & ho04 \\
\hline \multirow[t]{2}{*}{030226} & 1.986 & gr03b & 0.55 & 0.05 & 0.52 & 0.98 & 30.59 & $\mathrm{pa} 04$ \\
\hline & & & $0.70 \pm 0.03$ & & 0 & 0 & 30.27 & kl04 \\
\hline 030323 & 3.3718 & vr03b & $0.89 \pm 0.04$ & 0.13 & 0 & 0 & 30.92 & vr04 \\
\hline \multirow[t]{2}{*}{030329} & 0.1685 & $\mathrm{ca03}$ & 0.5 & 0.07 & $0.30 \pm 0.03$ & 0.29 & 30.45 & bl04 \\
\hline & & & $0.8 \pm 0.2$ & & 0.12 & 0.12 & 30.40 & $\mathrm{ma} 03$ \\
\hline 030429 & 2.66 & we03 & $0.36 \pm 0.12$ & 0.165 & $0.34 \pm 0.04$ & 0.99 & 30.78 & ja04 \\
\hline
\end{tabular}

References: me97: Metzger et al. (1997); ga98: Garcia et al. (1998); ku98: Kulkarni et al. (1998); wi99: Wijers et al. (1999); dj99: Djorgovski et al. (1999); hj02: Hjorth et al. (2002); dj98: Djorgovski et al. (1998); bl98: Bloom et al. (1998); ke99: Kelson et al. (1999); vr99: Vreeswijck et al. (1999); ho00: Holland et al. (2000); vr99b: Vreeswijck et al. (1999) (GCN324); be99: Beuermann et al. (1999); vr99c: Vreeswijck et al. (1999) (GCN496); ga00: Garnavich et al. (2000); bl00: Bloom et al. (2000); fe00: Feng et al. (2000); je01: Jensen et al. (2001); k100: Klose et al. (2000); pr02: Price et al. (2002); ma05: Masetti et al. (2005); fy00: Fynbo et al. (2000); fy01: Fynbo et al. (2001); jh01b: Jha et al. (2001) (GCN974); st01: Stanek et al. (2001); jh01: Jha et al. (2001); le01: Lee et al. (2001); pr02a: Price et al. (2002); dj01: Djorgovski et al. (2001); gr03: Greiner et al. (2003); pr02b: Price et al. (2002); in01: Infante et al. (2001); ga03: Garnavich et al. (2003); gl01: Gladders et al. (2001); ja03: Jakobsson et al. (2003); ho02: Holland et al. (2002); at05: Atteia et al. (2005); hj03: Hjorth et al. (2003); ma02: Masetti et al. (2002); be03: Bersier et al. (2003); co03: fi02: Iore et al. (2002); Covino et al. (2003); gi02: Giannini et al. (2002); pa03: Pandey et al. (2003); vr03: Vreeswijck et al. (2003) (GCN1785); fo03: Fox et al. (2003); ho04: Holland et al. (2004); vr03b: Vreeswijck et al. (2003) (GCN1953) gr03b: Greiner et al. (2003) (GCN1886); vr04: Vreeswijk et al. (2004); ca03: Caldwell et al. (2003); pa04: Pandey et al. (2004); kl04: Klose et al. (2004); bl04: Bloom et al. (2004); ma03: Matheson et al. (2003); we03: Weidinger et al. (2003); ja04: Jakobsson et al. (2004).

Fig. 5. Without this correction, the optical luminosity distribution has a width of $\sigma \sim 0.39$ (see Table 2). This is partly due to those GRBs at high redshift for which even a moderate value of $A_{V}^{\text {host }}$ implies a relatively large absorption at the rest-frame frequency $v_{R}(1+z)$.

\section{Light curves of the optical luminosities}

In Fig. 4 we show the light curves in terms of the optical luminosities in the $R$-band as a function of the rest-frame time. As can be seen, there is a clear clustering of the light curves when corrected for the cosmological and extinction effects with respect to the light curves shown in Fig. 1. Most of the luminosities at
$12 \mathrm{~h}$ (since trigger) clusters around $\log L_{v_{R}} \sim 30.65$ (see Fig. 5). This is the main result of our paper. There are three exceptions, i.e. GRB 980613, GRB 011121, and GRB 021211, which stand out from the bulk of the other bursts by being underluminous by a factor $\sim 15$ with respect to the other GRBs.

Some of the light curves shown in Fig. 4 appear peculiar, in particular:

GRB 970508: the optical light curve of this burst showed an initial brightening followed, approximately at 1 day, by normal decay. For this reason we calculated $L_{v_{R}}$ at $12 \mathrm{~h}$ by extrapolating the light curve from the data above $\sim 30 \mathrm{~h}$ (rest frame). Choosing not to extrapolate from later times would 


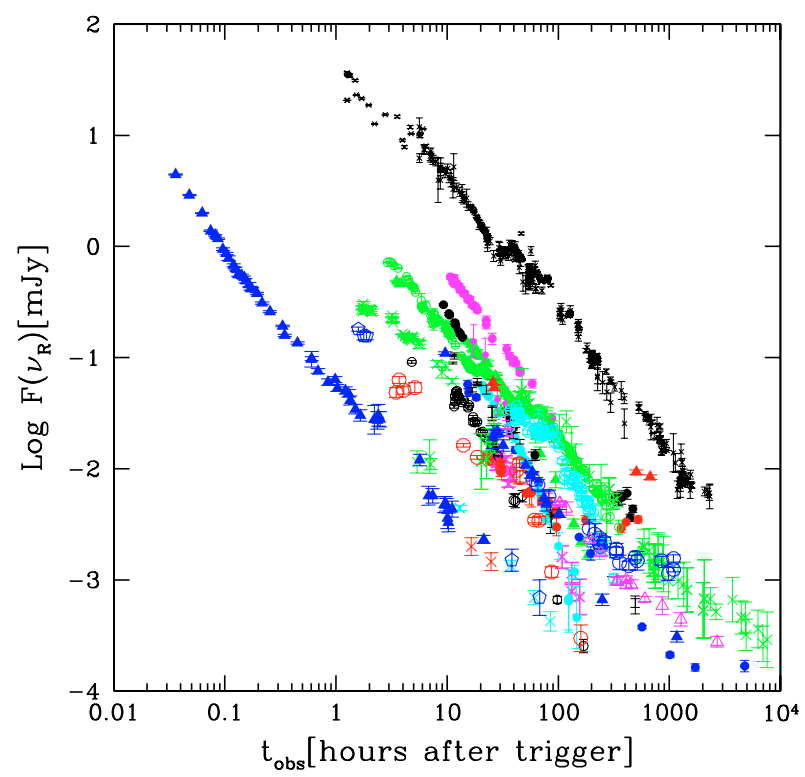

Fig. 1. Light curves in terms of observed fluxes versus observed time since the burst trigger for the 24 GRBs reported in Table 1. Fluxes have been corrected only for the galactic extinction. The references for all the plotted data are given in the appendix.

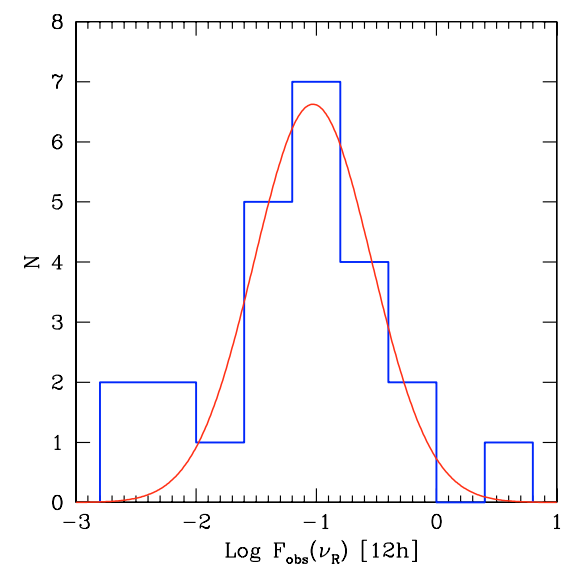

Fig. 2. Histogram of the observed fluxes (in $\mathrm{mJy}$ ) in the $R$-band (Cousin system) $12 \mathrm{~h}$ after the trigger (observer frame - from Fig. 1). All fluxes have been corrected for the foreground galactic extinction only. Superimposed to the histogram is a Gaussian fit to the data with mean value $\mu=-1.03$ and dispersion $\sigma=0.48$. Note that the fit is rather poor and most likely underestimates the real width of the distribution.

make this burst to belong to the "underluminous family" for times earlier than $12 \mathrm{~h}$.

GRB 020813 and GRB 030329: these GRB have an early jetbreak time (roughly at 4.6 and $10 \mathrm{~h}$, rest frame, respectively), and we calculated the $12 \mathrm{~h}$ luminosity by extrapolating from the light curve before $t_{\text {jet }}$. Choosing not to extrapolate from earlier times makes these bursts to remain in the same "luminous burst family".

As for the light curves of the observed fluxes, we can derive the distribution of the monochromatic optical luminosities at $12 \mathrm{~h}$ (rest frame) for the bursts of Fig. 4. This is shown in Fig. 5. The 24 GRBs separate into two groups: the bulk of GRBs (21 objects) with a $12 \mathrm{~h}$ rest frame luminosity distribution spanning less than one order of magnitude, and a second group (3 objects) that appears underluminous by a factor $\sim 15$. The first distribution can be represented well by a Gaussian with an average
Table 2. Distribution width of different quantities, according to a Gaussian fit.

\begin{tabular}{ll}
\hline \hline Distribution & $\sigma$ \\
\hline $\log L\left(v_{R}\right) @ 12 \mathrm{~h}$ rest frame & $0.28^{a}$ \\
$\log L_{\mathrm{X}}[4-20 \mathrm{keV}]$, $12 \mathrm{~h}$ rest frame & $0.74^{b}$ \\
$\log F\left(v_{R}\right) @ 12 \mathrm{~h}$ obs frame & 0.48 \\
$\log L\left(v_{R}\right) @ 12 \mathrm{~h}$ rest frame, no $A_{V}^{\text {host }}$ & $0.39^{a, c}$ \\
$\log E_{\gamma, \text { iso }}$ & 0.80 \\
$\log \left[v_{R} F\left(v_{R}\right) t_{12 \mathrm{~h}} /\right.$ Fluence $\left._{\gamma, \text { iso }}\right]$ & 0.93 \\
$\log \left[v_{R} L\left(v_{R}\right) t_{12 \mathrm{~h}} / E_{\gamma, \text { iso }}\right]$ & 0.9 \\
\hline
\end{tabular}

${ }^{a}$ Considering all bursts but the 3 underluminous ones; ${ }^{b}$ formal result from the fit, but the fit is poor; ${ }^{c}$ optical luminosities dereddened only for Galactic absorption; no host galaxy extinction has been considered.

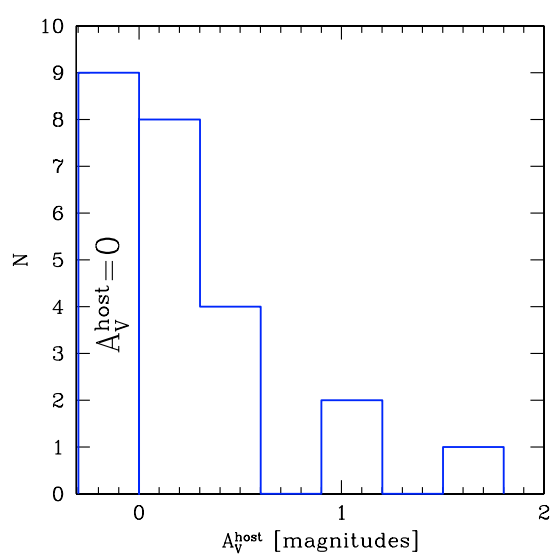

Fig. 3. Histogram of the host absorption values $A_{V}^{\text {host }}$ for the 24 GRBs of Table 1. The 9 GRBs in the first bin have an optical spectrum consistent with a null host-galaxy absorption.

luminosity $\left\langle\log L\left(v_{R}, 12 \mathrm{~h}\right)\right\rangle=30.65$ and a dispersion $\sigma=0.28$. The typical error on $\log L\left(v_{R}, 12 \mathrm{~h}\right)$ is around 0.1 , much less than the $1 \sigma$ dispersion of the distribution of this quantity. This error was estimated by propagating the average error on the observed magnitude $(0.1), A_{V}^{\text {host }}(0.13)$ and $\beta(0.1)$.

Boër \& Gendre (2000) have analyzed the behavior of the optical afterglow of the bursts studied in their paper ( 8 in total), without applying the dereddening of the extinction of the host (at that time largely unavailable). They did not find any clustering, nor a dichotomy, although (even without correcting for the absorption of the host), they noted that the distribution of the intrinsic optical luminosities was narrower than the distribution of the observed fluxes. The choice of $12 \mathrm{~h}$ rest frame is not critical for our results, as can be seen in Fig. 4, as long as the chosen time is less than the jet break time for most bursts.

Our result is surprising in many respects, as mentioned in the introduction. We can compare this narrow clustering of the optical luminosity with the distribution of the prompt-emission isotropic energy $E_{\gamma \text {,iso }}$ for the same bursts (see Table 4). The $\log E_{\gamma, \text { iso }}$ distribution (if fitted with a Gaussian) has a much larger dispersion of $\sigma \sim 0.8$ (see Table 2). Another unexpected result concerns the distribution of the ratio of $\log \left[L\left(v_{R}, 12 \mathrm{~h}\right) / E_{\gamma, \text { iso }}\right]$. Since we expect that the afterglow luminosity depends upon the isotropic kinetic energy of the fireball, which should be measured by $E_{\gamma, \text { iso }}$, we naively expect this distribution to have a smaller dispersion than either the $\log L\left(v_{R}\right)$ or the $\log E_{\gamma, \text { iso }}$ distribution. Instead, the $\log \left[L\left(v_{R}, 12 \mathrm{~h}\right) / E_{\gamma, \text { iso }}\right]$ distribution has a dispersion $\sigma=0.9$, even larger than the dispersion of $\log E_{\gamma \text {,iso }}$.

The three underluminous bursts, (i.e. GRB 980613, 011121, 021211) that seem to form a separate "family" are more than 


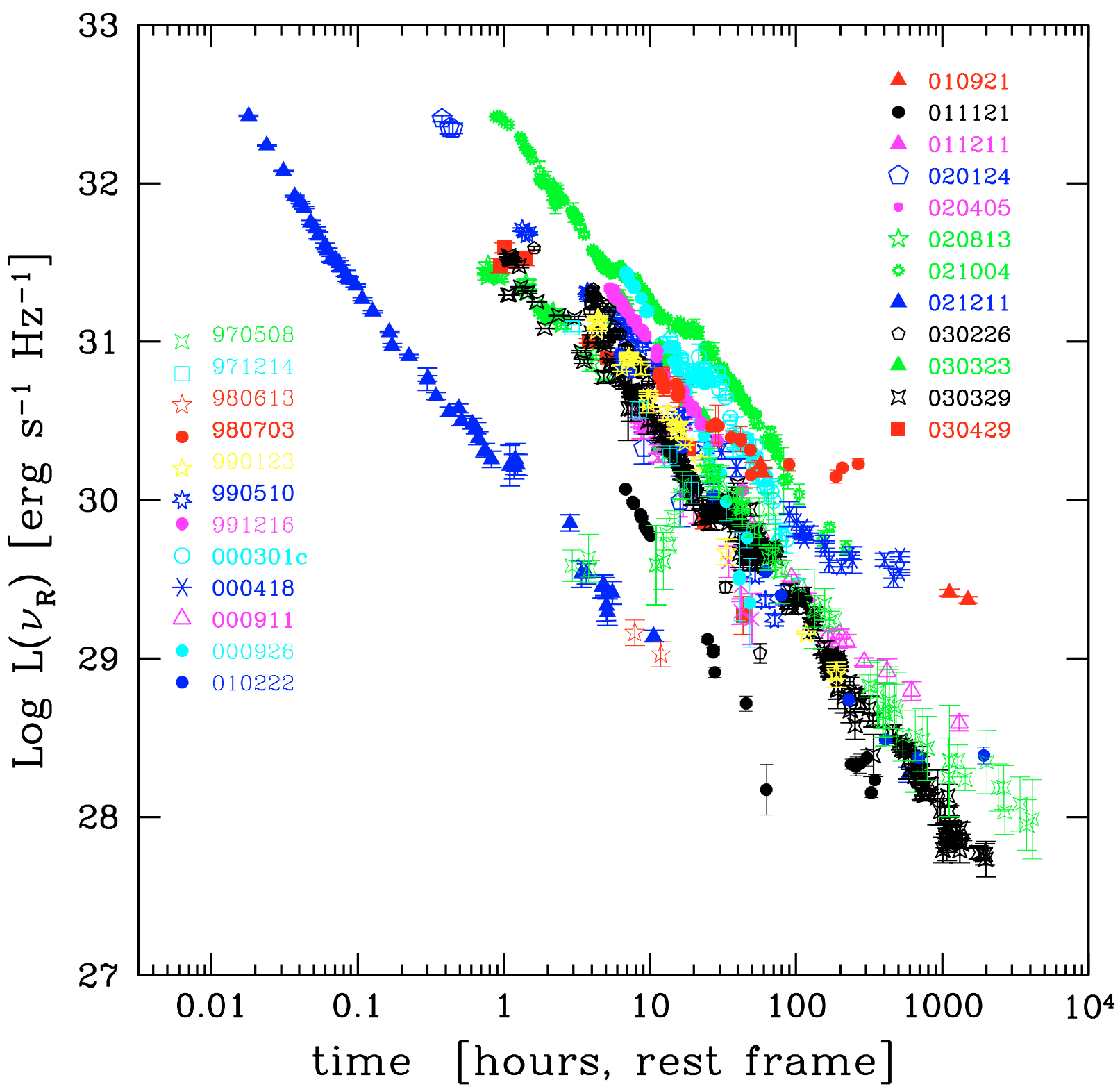

Fig. 4. Light curves of the optical luminosities as a function of the rest-frame time. The three underluminous GRBs are labelled. All data have been corrected for extinction (both Galactic and host). The references for the observed magnitudes can be found in the appendix. The references for the values of the spectral index $\beta$ and the host galaxy absorption can be found in the footnote of Table 1 .

$4 \sigma$ dimmer than the majority of bursts. For GRB 011121 and GRB 021211 the two parameters $\beta_{\mathrm{o}}$ and $A_{V}^{\text {host }}$ have possible different estimates (see Table 1). Here we adopted the values reported in the first line of Table 1 . However, if we consider the other possible choices, the implied $L\left(v_{R}, 12 \mathrm{~h}\right)$ would be even smaller, making these two bursts even more inconsistent (more than $4.5 \sigma$ ) with the distribution of the bulk of the other GRBs. Instead, concerning the GRBs with different estimates of $\beta_{\mathrm{o}}$ and $A_{V}^{\text {host }}$ that fall in the more populated group, using the other choices would shift their luminosities by less than one $\sigma$ (except for GRB 980703, for which the shift would be $1.7 \sigma$ ).

The three underluminous bursts do not seem to have any distinguishing property other than their smaller optical luminosities: all three have "normal" optical decays, spectral indices and extinction values. However, 2 of these GRBs lie on the faint portion of the X-ray luminosity distribution, see Fig. 6 (for the third there is no X-ray detected afterglow). On the other hand, the faint end of the X-ray luminosity distribution also contains bursts that belong to the "bright" optical family (i.e. GRB 011211 and GRB 030326). GRB 000210 and GRB 000214, lying at the faint extreme of the X-ray luminosity distribution, are dark bursts.

\subsection{Comparison with $X$-ray afterglow luminosities}

When studying the X-ray afterglow light curves, GB05 found that the distribution of the rest frame [4-20] $\mathrm{keV}^{3} \mathrm{X}$-ray luminosities is bimodal, clustering around two values. We expanded the original list of GB05 by including of three more GRBs: GRB 020405, GRB 020813, GRB 021004. We also slightly modified some of the data presented in their original table, as new information is now available for some of the bursts. For this reason we have collected the information about the X-ray data in

3 The light curves plotted in their Fig. 2 refer to the [2-10] keV band, but they locate all bursts at $z=1$. Therefore the rest frame energy band is $4-20 \mathrm{keV}$. 
Table 3. X-ray properties of the GRBs with known redshift and data collected from the literature. $\alpha$ and $\beta_{\mathrm{X}}$ are the temporal and spectral power law indices, respectively [i.e. $F(v, t) \propto t^{-\alpha} v^{-\beta_{\mathrm{X}}}$ ]. $F_{\mathrm{X}}$ is the observed X-ray flux integrated in the reported energy band and $L_{10}^{12 \mathrm{~h}}$ is the monochromatic $\mathrm{X}$-ray luminosity at $12 \mathrm{~h}$ (rest frame) calculated at $10 \mathrm{keV}$.

\begin{tabular}{|c|c|c|c|c|c|c|c|c|}
\hline$\overline{\text { GRB }}$ & $z$ & $\alpha$ & $\beta_{\mathrm{X}}$ & $\begin{array}{c}F_{\mathrm{X}} \\
10^{-12} \mathrm{cgs}\end{array}$ & $t_{\mathrm{obs}}$ & $\begin{array}{l}\text { band } \\
\mathrm{keV}\end{array}$ & $\log L_{10 \mathrm{keV}}^{12 \mathrm{~h}}$ & Ref. \\
\hline 970228 & $0.695^{1}$ & $1.3 \pm 0.1$ & $1.1 \pm 0.3$ & $1.0 \pm 0.2$ & $1 \mathrm{~d}$ & $2-10$ & 26.81 & $\operatorname{co9} 97$ \\
\hline 970508 & 0.835 & $1.1 \pm 0.1^{a}$ & $1.1 \pm 0.3$ & $1.0 \pm 0.4$ & $1 d$ & $2-10$ & 26.97 & gb05, pi98 \\
\hline 971214 & 3.418 & $1.1 \pm 0.1$ & $1.2 \pm 0.4$ & $0.23 \pm 0.05$ & $1 d$ & $2-10$ & 27.47 & co99, gb05 \\
\hline 980613 & 1.096 & $1.1 \pm 0.2$ & 1 & $0.27 \pm 0.07$ & $1 d$ & $2-10$ & 26.63 & $\operatorname{co} 99$ \\
\hline 980703 & 0.966 & $0.9 \pm 0.2$ & $1.8 \pm 0.4$ & $0.48 \pm 0.07$ & $1 d$ & $2-10$ & 26.70 & gb05 \\
\hline \multirow[t]{2}{*}{990123} & 1.60 & 1.35 & $0.99 \pm 0.07$ & $5.3 \pm 0.2$ & $11 \mathrm{~h}$ & $1.6-10$ & 27.69 & dp03, he99 \\
\hline & & $1.44 \pm 0.11$ & $1.00 \pm 0.05$ & $1.8 \pm 0.4$ & $1 \mathrm{~d}$ & $2-10$ & 27.72 & gb05 \\
\hline 990510 & 1.619 & $1.4 \pm 0.1$ & $1.2 \pm 0.2$ & $1.2 \pm 0.2$ & $1 \mathrm{~d}$ & $2-10$ & 27.57 & gb05 \\
\hline \multirow[t]{2}{*}{991216} & 1.02 & $1.6 \pm 0.1$ & $1.2 \pm 0.2$ & 2.58 & $37 \mathrm{~h}$ & $2-10$ & 27.77 & pi00 \\
\hline & & $1.6 \pm 0.1$ & $0.8 \pm 0.5$ & $5.6 \pm 0.3$ & $1 \mathrm{~d}$ & $2-10$ & 27.89 & gb05 \\
\hline \multirow[t]{2}{*}{000210} & $0.846^{2}$ & $1.38 \pm 0.03$ & $0.95 \pm 0.15$ & $0.4 \pm 0.06$ & $11 \mathrm{~h}$ & $2-10$ & 26.13 & pi02 \\
\hline & & $1.38 \pm 0.03$ & $0.9 \pm 0.2$ & $0.21 \pm 0.06$ & $1 d$ & $2-10$ & 26.32 & gb05 \\
\hline \multirow[t]{2}{*}{000214} & $0.42^{3}$ & $0.8 \pm 0.5^{b}$ & $1.2 \pm 0.3$ & $0.77 \pm 0.08$ & $15 \mathrm{~h}$ & $2-10$ & 26.01 & an00 \\
\hline & & $0.7 \pm 0.3$ & $1.2 \pm 0.5$ & $0.6 \pm 0.2$ & $1 \mathrm{~d}$ & $2-10$ & 26.05 & gb05 \\
\hline 000926 & 2.066 & $1.7 \pm 0.5$ & $0.7 \pm 0.2$ & $0.12 \pm 0.1$ & $2.78 \mathrm{~d}$ & $2-10$ & 27.35 & gb05 \\
\hline 010222 & 1.477 & $1.33 \pm 0.04$ & $1.01 \pm 0.06$ & $2.7 \pm 0.6$ & $1 \mathrm{~d}$ & $2-10$ & 27.85 & gb05 \\
\hline 011121 & 0.36 & $4_{-2}^{+3}$ & $2.4 \pm 0.4$ & $0.6 \pm 0.2$ & $1 d$ & $2-10$ & 26.11 & gb05 \\
\hline 011211 & 2.14 & $1.3 \pm 0.1$ & $1.2 \pm 0.1$ & $0.03 \pm 0.01$ & $1 d$ & $2-10$ & 26.19 & gb05 \\
\hline 020405 & 0.69 & $1.9 \pm 1.1$ & $0.72 \pm 0.21$ & $1.36 \pm 0.25$ & $1.71 \mathrm{~d}$ & $0.2-10$ & 27.21 & $\mathrm{mi03}$ \\
\hline 020813 & 1.25 & $1.38 \pm 0.06$ & $0.85 \pm 0.04$ & 2.2 & $1.33 \mathrm{~d}$ & $0.6-6$ & 27.70 & bu03 \\
\hline 021004 & 2.33 & $0.9 \pm 0.1$ & $1.01 \pm 0.08$ & 0.63 & $1.37 \mathrm{~d}$ & $0.6-6$ & 27.60 & but03 \\
\hline 030226 & 1.98 & $2.7 \pm 1.6$ & $0.9 \pm 0.2$ & $0.035 \pm 0.002$ & $1.77 \mathrm{~d}$ & $2-10$ & 26.59 & gb05 \\
\hline 030329 & 0.168 & $0.9 \pm 0.3$ & $0.9 \pm 0.2$ & $14.3 \pm 2.9$ & $1 \mathrm{~d}$ & $2-10$ & 26.69 & gb05 \\
\hline
\end{tabular}

${ }^{a} 970508$ showed a substantial rebrightening, correlated with the optical (Piro et al. 1998); ${ }^{b}$ decay index considering only MECS data. If WFC is included, $\alpha=1.41 \pm 0.03$ (Antonelli et al. 2000). For GRB 030329 we have calculated the X-ray flux at $12 \mathrm{~h}$ rest frame extrapolating from earlier data, since this GRBs showed a jet break at approximately $10 \mathrm{~h}$ (rest frame time) (see Tiengo et al. 2004). References: co97: Costa et al. (1997); gb05: Gendre \& Boër (2005); pi98: Piro et al. (1998); st04: Stratta et al. (2004); co99: Costa et al. (1999); dp03: De Pasquale et al. (2003); he99: Heise et al. (1999); pi00: Piro et al. (2000); pi02: Piro et al. (2002); an00: Antonelli et al. (2000); mi03: Mirabal et al. (2003); bu03: Butler et al. (2003). ${ }^{1}$ Djorgovski et al. (1999); ${ }^{2}$ Piro et al. (2002); ${ }^{3}$ Antonelli et al. (2000).

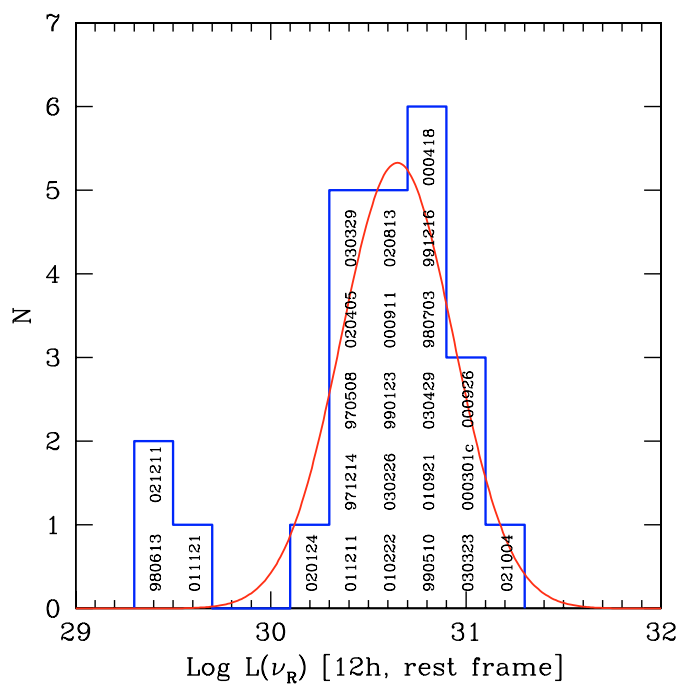

Fig. 5. Histogram of the monochromatic optical luminosities $12 \mathrm{~h}$ (rest frame) after the trigger for the 24 GRBs reported in Table 1 and shown in Fig. 4. Data have been dereddened both for Galactic and host extinction. The solid red line represents the Gaussian fit to the data with mean value $\mu=30.65$ and dispersion $\sigma=0.28$.

Table 3, with the appropriate references. We find a more continuous distribution than do GB05, as can be seen in Fig. 6, without clear clustering or a clear separation in two GRB "families".

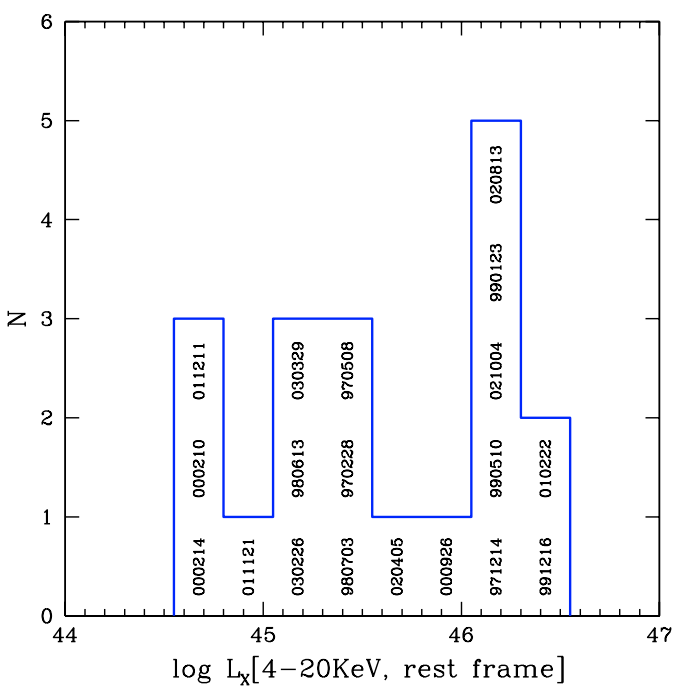

Fig. 6. Histogram of the X-ray luminosities $12 \mathrm{~h}$ (rest frame) after the trigger, calculated in the rest frame band [4-20 keV].

Figure 6 shows that the distribution of X-ray luminosities is wider than the distribution of the optical luminosities. A Gaussian fit (although poor) gives a dispersion $\sigma=0.74$ (see Table 2). Figure 7 shows the monochromatic [2 keV, rest frame] $\mathrm{X}$-ray luminosity as a function of the optical monochromatic luminosity [ $R$-band] $12 \mathrm{~h}$ after trigger, in the rest frame. Dashed diagonal lines correspond to lines of constant broad band spectral 


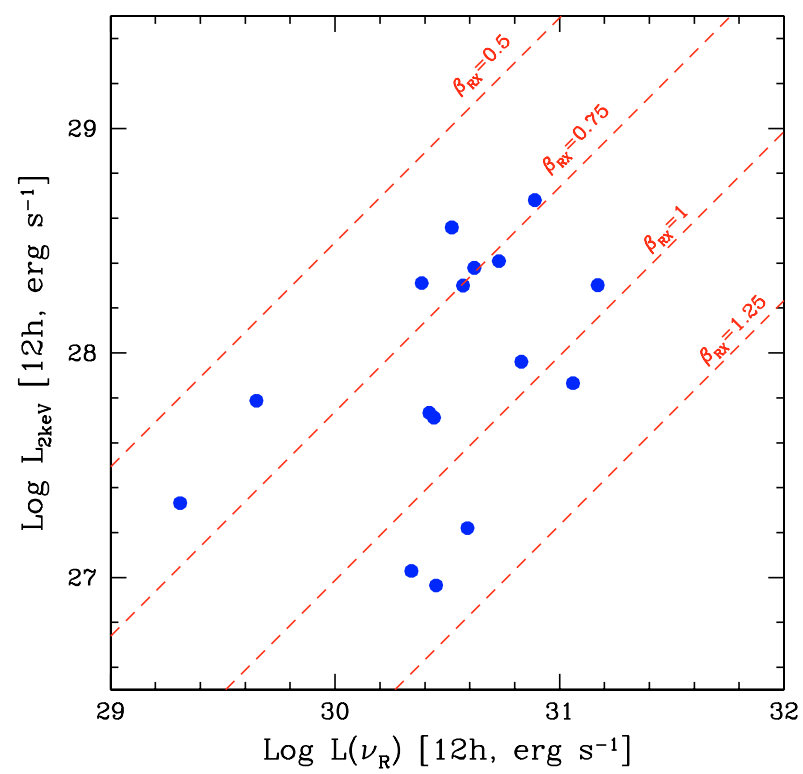

Fig. 7. X-ray monochromatic [2 keV, rest frame] luminosity as a function of the optical monochromatic [ $R$ band, rest frame] luminosity at $12 \mathrm{~h}$ after trigger. The dashed lines correspond to different broad-band spectral indices $\beta_{\mathrm{RX}}$ as labelled.

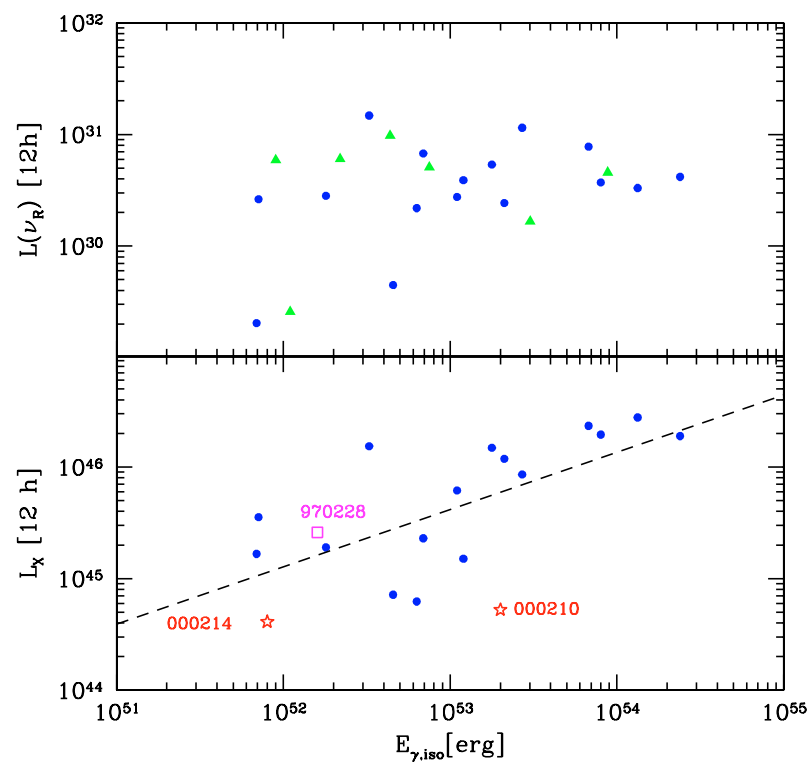

Fig. 8. Optical monochromatic luminosity (top panel) and X-ray [4-20 keV] luminosities (mid panel) at $12 \mathrm{~h}$ after trigger (rest frame time) as a function of the isotropic emitted energy during the prompt phase (integrated between $1 \mathrm{keV}$ and $10 \mathrm{MeV}$, see Ghirlanda et al. 2004). Circles correspond to bursts having both optical and X-ray data. Triangles are GRBs with optical but no X-ray data. Stars are the two GRBs (as labelled) with X-rays but no optical data. We also show GRB 970228, for which there is no information on the amount of extinction in the host (square). The dashed line is the linear regression fit $\left(\log L_{\mathrm{x}} \propto 0.51 \log E_{\gamma, \text { iso }}\right)$, which has a chance probability $P=3 \times 10^{-3}$.

indices $\beta_{R X}$ between the optical ( $R$-band) and the X-ray ( $\left.2 \mathrm{keV}\right)$, defined as

$\beta_{R X}=\frac{\log \left(L_{v_{R}} / L_{\mathrm{X}}\right)}{\log \left(v_{\mathrm{X}} / v_{R}\right)}$.

This figure shows how bursts that are more luminous in $\mathrm{X}$ rays tend to have flatter $\beta_{R \mathrm{X}}$ spectral indices (and therefore are

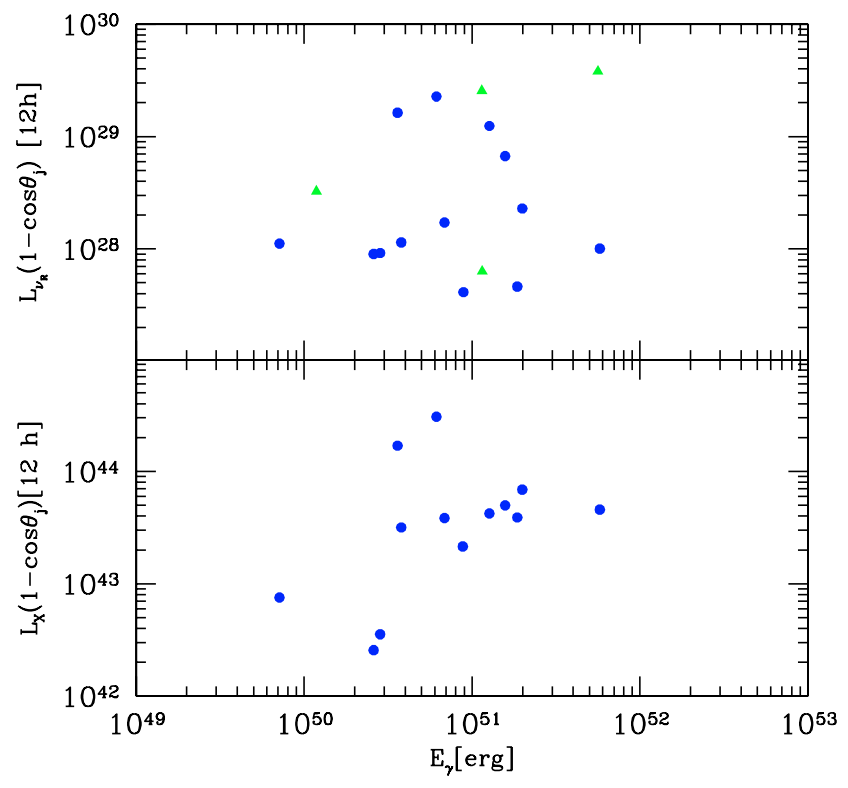

Fig. 9. For those bursts of measured $\theta_{\mathrm{j}}$, we have calculated the collimation corrected afterglow luminosity (top panel: optical; bottom panel: $\mathrm{X}$-ray) at $12 \mathrm{~h}$ after trigger (rest-frame time) as a function of the collimation-corrected emitted energy during the prompt phase (integrated between $1 \mathrm{keV}$ and $10 \mathrm{MeV}$, see Ghirlanda et al. 2004). Symbols as in Fig. 8. There is now no correlation between the optical or the X-ray luminosity and the prompt emitted energy.

relatively less luminous in the optical) and viceversa. There are two exceptions, both belonging to the dim optical family. This behavior (flatter $\beta_{R \mathrm{X}}$ for greater $L_{\mathrm{X}}$ ) is a necessary condition for having optical luminosities that are more clustered than the $\mathrm{X}$-ray ones.

\subsection{Comparison with the emitted $\gamma$-ray energies}

Figure 8 shows both the optical and X-ray luminosities as a function of $E_{\gamma, \text { iso }}$, the isotropic energy emitted during the prompt phase. There is no correlation in the case of the optical luminosities (top panel), while there is some correlation in the case of X-ray luminosities, albeit not very strong (chance probability $\left.P \sim 3 \times 10^{-3}\right)$. Both quantities $\left(L_{\mathrm{x}}\right.$ and $\left.E_{\gamma, \text { iso }}\right)$, being isotropic, depend on the aperture angle of the jet $\theta_{\mathrm{j}}$. For those GRBs for which we know $\theta_{\mathrm{j}}$ (13 objects), we can construct the collimation corrected quantities by multiplying by $\left(1-\cos \theta_{\mathrm{j}}\right)$. After doing that, the correlation between the X-ray luminosity and the $\gamma$-ray energy disappears (bottom panel of Fig. 9).

We would have expected the same effect in the case of the optical luminosities; i.e. there should be an apparent correlation when considering isotropic quantities, but there is not (upper panel of Fig. 8). Furthermore, the distribution of the collimationcorrected optical luminosities becomes not narrower, but slightly broader than the distribution of the isotropic luminosities, as can be seen from the upper panel of Fig. 9, which also shows that there is no correlation between the collimation-corrected optical luminosities and prompt emitted energies. We have also verified that there is no correlation between the optical or the X-ray luminosity and the spectral peak energy $E_{\text {peak }}$ of the prompt emission.

\section{Spectral energy distributions}

Figures 10 and 11 show the optical to X-ray spectral energy distribution (SED) for all bursts in our sample. Data are 


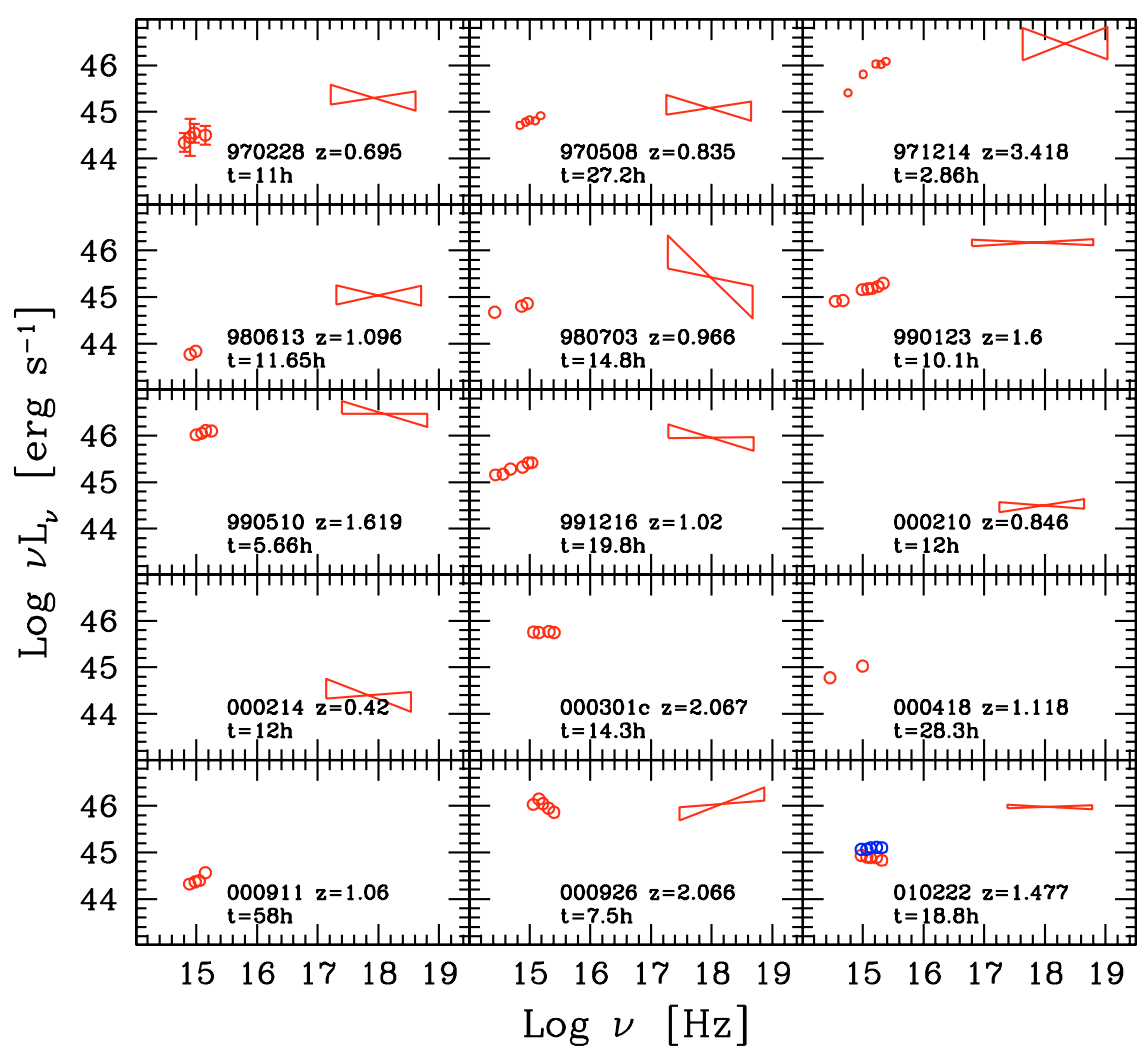

Fig. 10. Optical to X-ray spectral energy distribution for all GRBs in our sample. Data are simultaneous, at the rest-frame time labelled in each panel. Sources of data: GRB 970228: optical: Reichart (1999); X-rays: Costa et al. (1997). GRB 970508: Galama et al. (1998); Piro et al. (1998). GRB 971214: Wijers et al. (1999); Stratta et al. (2004). GRB 980613: Hjorth et al. (2002); Costa (1999). GRB 980703: Vreeswijk et al. (1999); De Pasquale et al. (2003). GRB 990123: Galama et al. (1999); Heise et al. (1999). GRB 990510: optical: Harrison et al. (1999); X-rays: Gendre \& Boër (2005). GRB 991216: Halpern et al. (2000), Garnavich et al. (2000); Piro et al. (2000). GRB 000210: Piro et al. (2002). GRB 000214: Antonelli et al. (2000). GRB 000301c: Jensen et al. (2001). GRB 000418: Klose et al. (2000). GRB 000911: Masetti et al. (2005). GRB 000926: optical: Fynbo et al. (2001). X-rays: Gendre \& Boër (2005). GRB 010222: optical: Masetti et al. (2001). X-rays: Gendre \& Boër (2005).

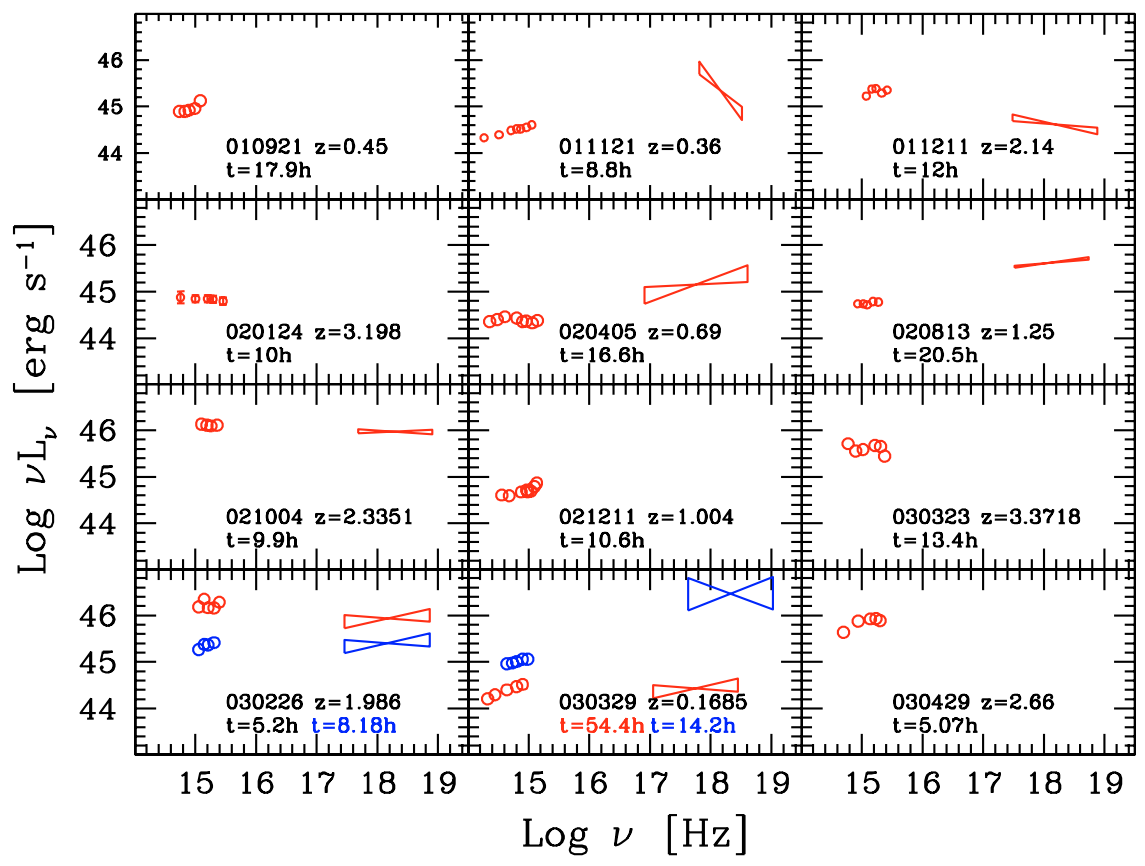

Fig. 11. Optical to X-ray spectral energy distribution for all GRBs in our sample. Data are simultaneous, at the rest-frame time labelled in each panel. Sources of data: GRB 010921: Price et al. (2002). GRB 011121: optical: Garnavich et al. (2003); X-rays: Gendre \& Boër (2005). GRB 020124: Hjorth et al. (2003). GRB 020405: Masetti et al. (2003); Mirabal et al. (2003). GRB 020813: optical: Covino et al. (2003); X-rays: Butler et al. (2003). GRB 021004: optical: Pandey et al. (2003); X-rays: Butler et al. (2003). GRB 021211: Nysewander et al. (2005). GRB 030323: Vreeswijk et al. (2004). GRB 030226: optical: Pandey et al. (2004); X-rays: Gendre \& Boër (2005). GRB 030329: optical: Bloom et al. (2004); Matheson et al. (2003); X-rays: Gendre \& Boër (2005). GRB 030429: Jakobsson et al. (2004). 
plotted in the rest frame of the source, after being corrected for extinction. In constructing the SED we considered the optical multiband photometry at as consistent a time as possible with the X-ray observations, requiring the least possible extrapolation from data taken at other times. In some cases (i.e. GRB 030226 and GRB 030329), we plotted two SED for each burst corresponding to two different observing times or, in the case of GRB 010222, corresponding to two different choices of hostgalaxy optical extinction. The captions of these figures report the original sources of data.

This figure shows that in a large fraction of cases the optical to X-ray data seem to be consistent with being produced by the same emission process. In a minority of cases (GRB 000926, possibly GRB 020405, and the earlier SED of GRB 030226), the optical spectrum is steeper than the X-ray spectrum, suggesting that they are produced by a different component. One obvious possibility is the inverse Compton process dominating the X-ray flux at the observing times. There is finally one case (GRB 020813) where the optical and the X-ray emission smoothly join, but the peak of the overall SED lies above the X-ray band. To summarize:

- Of the 27 SED of GRBs shown, 17 have both optical and X-rays (note that we now include GRB 970228), 2 have only the X-ray data (GRB 000210 and GRB 000214), and 8 have only the optical data (GRB 000301c, GRB 000418, GRB 000911, GRB 010921, GRB 020124, GRB 021211, GRB 030323, GRB 030429).

- In 13 out of 17 cases, the extrapolated optical and X-ray spectra join smoothly, indicating a common (synchrotron) origin by a population of electrons characterized by an energy break (flatter at low energies and steeper at high energies, as expected in the case of incomplete cooling).

- Of the remaining 4, GRB 000926 shows a steep optical and a flat X-ray spectrum, suggesting that the X-ray flux has a non-synchrotron origin. The same (but less extreme) behavior characterizes the SED of GRB 020405 and the early time SED of GRB 030226. The SED of GRB 010222 is somewhat difficult to classify, since the optical spectrum could smoothly join the X-ray one if the absorption is slightly underestimated. Note that GRB 000926 and GRB 010222 are the two bursts lying in between the two groups of X-ray luminosities identified by GB05.

- Of the 13 "normal synchrotron" SEDs, in 11 cases the $v L_{v}$ peak, $v_{\text {peak }}$, is between the optical and the X-ray band or in the X-ray band. The uncertain cases are due to the relatively large uncertainties of the X-ray slope, which are often characterized by a spectral index close to unity (i.e. flat in $v L_{v}$ ). In GRB 021004, $v_{\text {peak }}$ could be in the $I R$ band, but the overall spectrum is nearly flat in $v L_{v}$. In GRB 020813, $v_{\text {peak }}$ is above the $\mathrm{X}$-ray range.

\section{SWIFT bursts}

At the time of writing (November 2005), there are 18 long GRBs detected by SWIFT for which the redshift has been spectroscopically determined. We list them in Table 5, together with their redshifts, galactic extinction, and, when possible, the calculated optical luminosities (at $12 \mathrm{~h}$ rest frame). We also list the X-ray luminosities calculated in the (rest frame) 4-20 keV band, $12 \mathrm{~h}$ after trigger.

We alert the reader that for all these GRBs but GRB 050401, GRB 050525, and GRB 050730 there is no information yet about the optical extinction in the rest frame of the source. Therefore

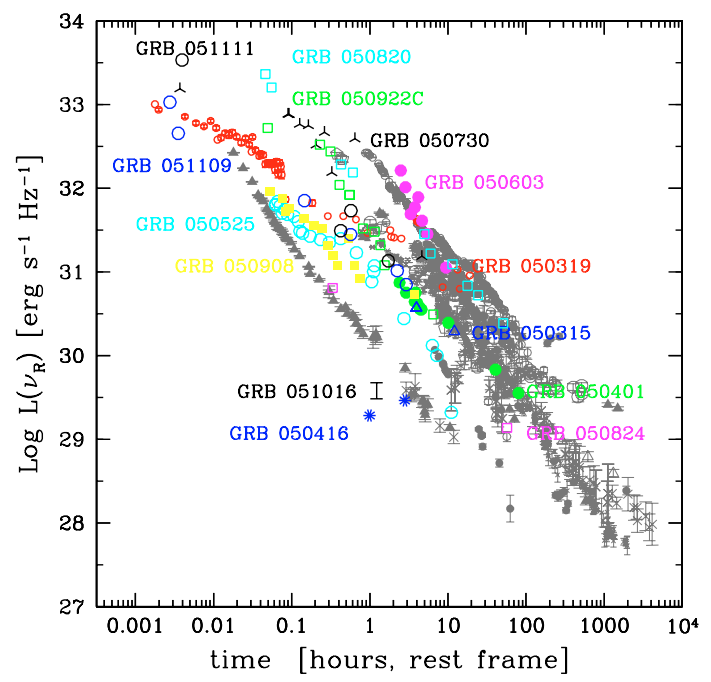

Fig. 12. Superposed light curves of the optical luminosities in the rest frame of the 14 GRBs detected by SWIFT with enough photometric data to the light curves already shown in Fig. 4 (grey dots). The luminosities of all SWIFT GRBs are corrected only for the galactic absorption, except for GRB 050401, for which $A_{V}^{\text {host }}=0.67$ (Watson et al. 2005), GRB 050525, for which $A_{V}^{\text {host }}=0.25 \pm 0.15$ (Blustin et al. 2005) and GRB 050730 for which $A_{V}^{\text {host }} \sim 0$ (Starling et al. 2005). The SWIFT GRBs luminosities have been k-corrected assuming a common spectral index $\beta=1$. The names of the SWIFT GRBs are given near their light curves.

the listed value of $L\left(v_{\mathrm{R}}\right)$ is not corrected for extinction in the rest frame and it is $\mathrm{k}$-corrected assuming $\beta=1$, except for GRB 050401, for which we used $A_{V}^{\text {host }}=0.67$ and $\beta=0.5$ (Watson et al. 2005) GRB 050525, for which we used the values given in Blustin et al. (2005). For GRB 050730 Starling et al. (2005) give a measured value of $\beta=1$. The optical light curves of the 14 SWIFT bursts with redshift are shown in Fig. 12, superposed to the light curves (green or light grey symbols) of the other bursts. For the remaining 4 SWIFT bursts with known redshift, we could not find enough information in the literature to plot their light curve. In Fig. 13 we show the histogram of the optical luminosities after the addition of the 14 SWIFT bursts for which we could calculate this quantity. As can be seen, although the behavior of the light curve of some of the SWIFT bursts is peculiar, their $L\left(v_{\mathrm{R}}\right)$ at $12 \mathrm{~h}$ is entirely consistent with the distribution found previously.

The X-ray luminosity distribution with the addition of these SWIFT bursts is shown in Fig. 14. The SWIFT bursts appear, on average, more luminous in X-rays than the pre-SWIFT bursts. This is probably due to the fact that the average redshift of SWIFT bursts is somewhat higher than the average redshift of the other bursts. $(\langle z\rangle=2.3 \pm 1.3$ for the bursts listed in Table 5 vs. $\langle z\rangle=1.5 \pm 0.9$ for the bursts listed in Table 4.)

We conclude that the indications coming from the first SWIFT bursts with known redshift strongly confirm the picture presented in this paper. Despite the difference in average redshift, and despite the broadening of the X-ray luminosity distribution, the clustering of the large majority of the optical luminosities is confirmed. In addition, the SWIFT bursts also confirm the existence of a dichotomy of the optical luminosity distribution, with the presence of an underluminous family. 


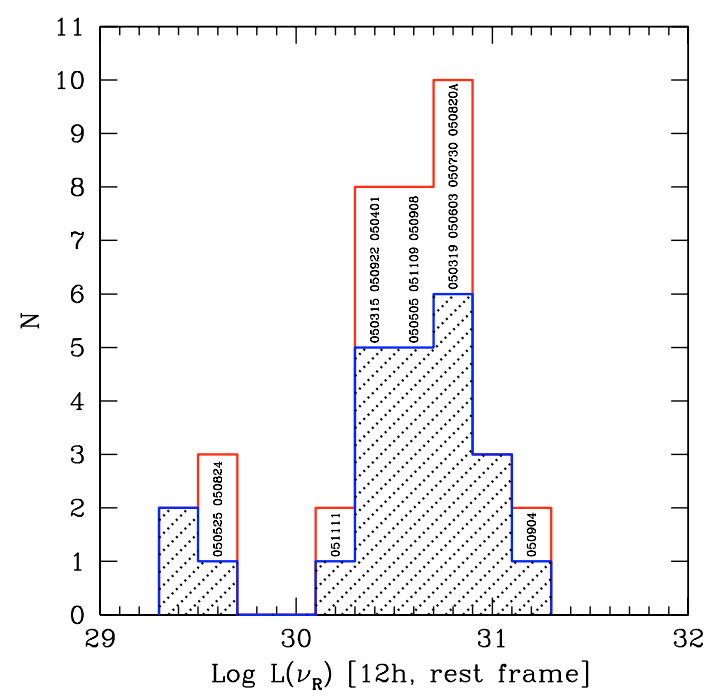

Fig. 13. Like Fig. 5 with added, the monochromatic optical luminosities $12 \mathrm{~h}$ (rest frame) after the trigger of the 14 GRBs discovered by SWIFT whose intrinsic luminosities could be calculated from the photometric data. We caution the reader that the optical luminosities of all SWI bursts (except GRB 050401, GRB 050525 and GRB 050730) are uncorrected for the host galaxy absorption and are $k$-corrected assuming a the same optical spectral index $(\beta=1)$ for all bursts (except GRB 050401, GRB 0505025 and GRB 050730).

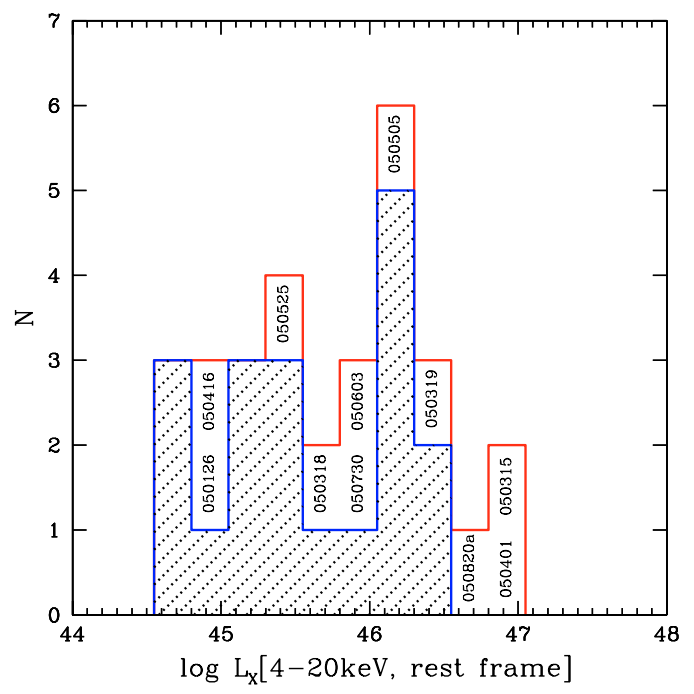

Fig. 14. Like Fig. 6, the X-ray [4-20 keV] luminosities $12 \mathrm{~h}$ (rest frame) after the trigger of the 11 GRBs discovered by SWIFT whose intrinsic luminosities could be calculated from the data found in Nousek et al. (2005; for GRB 050730, see Perri et al. 2005; for GRB 050820, see Page et al. 2005).

\section{Discussion}

The main result of our study is the clustering of the optical luminosities of the afterglows of GRBs. That is, bursts with widely different isotropic gamma-ray emitted energies are nevertheless similar in their optical output.

This result is unexpected for several reasons: i) the optical luminosities, for the time of interest, do not dominate the bolometric radiated output; ii) in contrast to the X-ray frequencies, likely to be above the cooling frequency $v_{\mathrm{c}}$, the optical frequencies are likely to be smaller than $v_{\mathrm{c}}$. This is confirmed by the simultaneous SED shown in Figs. 10 and 11. This implies that the optical emission does indeed depend on the density of the
Table 4. Values of redshift, $E_{\gamma \text {,iso }}$ and the semiaperture jet angle $\theta_{\mathrm{j}}$ used in Fig. 8, taken from Ghirlanda et al. (2004). When present, the values in parenthesis are the errors.

\begin{tabular}{llll}
\hline \hline GRB & $z$ & $\begin{array}{l}E_{\gamma, \text { iso }} \\
{[\mathrm{erg}]}\end{array}$ & $\begin{array}{l}\theta_{\mathrm{j}} \\
{[\mathrm{deg}]}\end{array}$ \\
\hline 970508 & 0.835 & $7.1 \mathrm{E} 51(0.15)$ & $24.0(3.3)$ \\
971214 & 3.418 & $2.11 \mathrm{E} 53(0.24)$ & $\ldots$ \\
980613 & 1.096 & $6.9 \mathrm{E} 51(0.95)$ & $\ldots$ \\
980703 & 0.966 & $6.9 \mathrm{E} 52(0.82)$ & $11(0.8)$ \\
990123 & 1.6 & $2.39 \mathrm{E} 54(0.28)$ & $3.98(0.57)$ \\
990510 & 1.616 & $1.78 \mathrm{E} 53(0.19)$ & $3.74(0.24)$ \\
991216 & 1.02 & $6.7 \mathrm{E} 53(0.81)$ & $4.4(0.6)$ \\
$000301 \mathrm{c}$ & 2.067 & $4.37 \mathrm{E} 52$ & 13.14 \\
000418 & 1.1181 & $7.51 \mathrm{E} 52$ & 22.3 \\
000911 & 1.06 & $8.8 \mathrm{E} 53(1.05)$ & $\ldots$ \\
000926 & 2.0375 & $2.7 \mathrm{E} 53$ & 6.19 \\
010222 & 1.477 & $1.33 \mathrm{E} 54(0.15)$ & $3.03(0.14)$ \\
010921 & 0.45 & $9.0 \mathrm{E} 51(1.0)$ & $\ldots$ \\
011121 & 0.36 & $4.55 \mathrm{E} 52(0.54)$ & $\ldots$ \\
011211 & 2.14 & $6.3 \mathrm{E} 52(0.7)$ & $5.2(0.63)$ \\
020124 & 3.198 & $3.02 \mathrm{E} 53(0.36)$ & $5.0(0.3)$ \\
020405 & 0.69 & $1.1 \mathrm{E} 53(0.13)$ & $6.4(1.05)$ \\
020813 & 1.25 & $8.0 \mathrm{E} 53(0.96)$ & $2.7(0.13)$ \\
021004 & 2.3351 & $3.27 \mathrm{e} 52(0.4)$ & $8.5(1.04)$ \\
021211 & 1.004 & $1.1 \mathrm{E} 52(0.13)$ & $\ldots$ \\
030226 & 1.986 & $1.2 \mathrm{E} 53(0.13)$ & $3.94(0.49)$ \\
030329 & 0.1685 & $1.8 \mathrm{E} 52(0.21)$ & $5.1(0.4)$ \\
030429 & 2.66 & $2.19 \mathrm{E} 52(0.26)$ & $5.96(1.43)$ \\
\hline
\end{tabular}

interstellar medium, $n$. A range in the values of $n$ should then contribute to increase the dispersion of the optical luminosities; iii) similar to the X-ray luminosities, $L_{\mathrm{opt}}$ also depends from the product of $E_{\mathrm{k} \text {,iso }}$ and a function on the equipartition parameters $\epsilon_{\mathrm{e}}$ and $\epsilon_{B}$. The observed clustering implies a corresponding "clustering" of the values of the isotropic kinetic energy and of the equipartition parameters.

\subsection{The "standard" external-shock synchrotron model}

In order to understand what has been observed, we should investigate the implications of these two facts: i) for the majority of bursts, $v_{\mathrm{c}}$ is between the optical and the X-ray band after a few hours (to a day) from trigger; ii) the distribution of the optical luminosities is narrower than the distribution of X-ray luminosities. Here we very briefly discuss these facts in the framework of the standard external shock synchrotron model.

As previously noted by Panaitescu \& Kumar (2000, 2001, 2002) having $v_{\mathrm{c}}$ between the optical and X-ray bands a day after the trigger implies a relatively low value of $\epsilon_{B}$ (and $n$ ). For convenience, we report here Eq. (27) (for homogeneous ISM density) and Eq. (28) (for a $r^{-2}$ wind profile) of Panaitescu \& Kumar (2000) for $v_{\mathrm{c}}$ :

$\begin{array}{ll}v_{\mathrm{c}}=3.7 \times 10^{14} E_{53}^{-1 / 2} n^{-1}(Y+1)^{-2} \epsilon_{B,-2}^{-3 / 2} t_{d}^{-1 / 2} & \mathrm{~Hz} \\ v_{\mathrm{c}}=3.4 \times 10^{14} E_{53}^{1 / 2} A_{*}^{-2}(Y+1)^{-2} \epsilon_{B,-2}^{-3 / 2} t_{d}^{1 / 2} & \mathrm{~Hz}\end{array}$

where the notation $Q=10^{x} Q_{x}$ is adopted. Here, $E$ is the isotropic kinetic energy of the fireball, $t_{\mathrm{d}}$ is the time after trigger measured in days and $Y$ is the Comptonization parameter. For the wind case, it is assumed that $n(r)=A r^{-2}$ and that $A_{*}$ is the value of $A$ when setting $\dot{M}=10^{-5} M_{\odot} \mathrm{yr}^{-1}$ and a wind velocity $v=10^{3} \mathrm{~km} \mathrm{~s}^{-1}$. From the above equations, values of $v_{\mathrm{c}}$ close to $10^{16} \mathrm{~Hz}$ require $\epsilon_{B} \sim 10^{-3}$ or less. The possible dependence 
Table 5. SWIFT long bursts with spectroscopically measured redshift.

\begin{tabular}{lllll}
\hline \hline GRB & $z$ & $A_{R}^{\text {Gal }}$ & $\log L\left(v_{R}\right)$ & $\log L_{\mathrm{X}}$ \\
\hline 050126 & 1.29 & 0.15 & & 44.8 \\
050315 & 1.949 & 0.13 & 30.33 & 47.0 \\
050318 & 1.44 & 0.046 & & 45.7 \\
050319 & 3.24 & 0.031 & 30.7 & 46.4 \\
050401 & 2.90 & 0.175 & $30.4^{a}$ & 46.9 \\
050416 & 0.654 & 0.13 & & 44.9 \\
050505 & 4.3 & 0.058 & 30.5 & 46.3 \\
050525 & 0.606 & 0.255 & $29.6^{a}$ & 45.4 \\
050603 & 2.821 & 0.074 & 30.8 & 46.0 \\
050730 & 3.967 & 0.135 & $30.79^{a}$ & 45.9 \\
$050820 \mathrm{~A}$ & 2.612 & 0.123 & 30.81 & 46.65 \\
050824 & 0.83 & 0.093 & 29.64 & \\
050904 & 6.29 & 0.161 & $31.18^{b}$ & \\
050908 & 3.3437 & 0.069 & 30.52 & \\
$050922 \mathrm{C}$ & 2.198 & 0.27 & 30.34 & \\
$051016 \mathrm{~B}$ & 0.9364 & 0.13 & & \\
051109 & 2.346 & 0.508 & 30.63 & \\
051111 & 1.55 & 0.43 & 30.24 & \\
\hline
\end{tabular}

a Corrected for the host galaxy absorpion and using the spectral index $\beta_{\mathrm{o}}$ found in litterature; ${ }^{b}$ extrapolated from $J$ band photometry. References: GRB 050315: Cobb et al. (2005), GCN 3104; Cobb et al. (2005), GCN 3110, $z$ : Kelson et al. (2005), GCN 3101. GRB 050319: Woźniak et al. (2005), z: Fynbo et al. (2005), GCN 3136; GRB 050401: McNaught et al. (2005), GCN 3163; D'Avanzo et al (2005), GCN 3171; Kahharov et al. (2005), GCN 3174; Misra et al. (2005), GCN 3175; Greco et al. (2005), GCN 3319; Watson et al. (2005), $z$ : Fynbo et al. (2005c), GCN 3176. GRB 050505: Rol et al. (2005), GCN 3372; Chapman et al. (2005), GCN 3375; Klotz et al. (2005), GCN 3403, $z$ : Berger et al. (2005), GCN 3368. GRB 050525: Blustin et al. (2005), $z$ : Foley et al. (2005), GCN 3483. GRB 050603: Brown et al. (2005), GCN 3549, z: Berger et al. (2005), GCN 3520. GRB 050730: Sota et al. (2005), GCN 3705; Holman et al. (2005), GCN 3716, Haislip et al. (2005), GCN 3719; Klotz et al. (2005), GCN 3720, z: Holman et al. (2005), GCN 3716. GRB 050820: Prochaska et al. (2005); Fox \& Cenko (2005), GCN 3829; Cenko \& Fox (2005), GCN 3834; Page et al. (2005), GCN 3837, $z$ : Ledoux et al. (2005), GCN 3860. GRB 050824: Gorosabel (2005), GCN 3865; Halpern, J., P., GCN 3907, z: Fynbo et al. (2005), GCN 3874. GRB 050904: Haislip et al. (2005), GCN 3914; Tagliaferri et al. (2005), z: Kawai et al. (2005), GCN 3937. GRB 050908: Torii (2005), GCN 3943; Kirschbrown (2005), GCN 3947, Foley (2005), GCN 3949; Durig (2005), GCN 3950, z: Fugazza et al. (2005), GCN 3948. GRB 050922C: Fynbo (2005), GCN 4040; Durig (2005), GCN 4023; Covino (2005), GCN 4046, $z$ : D'Elia et al. (2005), GCN 4044; GRB 051109: Mirabal et al. (2005), GCN 4215; Milne et al. (2005), GCN 4218; Jelinek et al. (2005), GCN 4227; Huang et al. (2005), $z$ : Quimby et al. (2005), GCN 4221. GRB 051111: Mundell et al. (2005), GCN 4250; Milne et al. (2005), GCN 4252; Garimella et al. (2005), GCN 4257; Huang et al. (2005), GCN 4258; Cameron et al. (2005), GCN 4266, z: Hill et al. (2005), GCN 4255.

of $v_{\mathrm{c}}$ on the slope of the electron energy distribution is hidden in the $(Y+1)$ term. This term is important if $\epsilon_{B}$ is below some critical value (see discussion in Panaitescu \& Kumar 2000).

In order to find the simplest possible reason for the clustering of the optical luminosities, we again used the analytical prescriptions of Panaitescu \& Kumar (2000) to construct light curves and spectra at a given time. Figure 15 shows some examples of spectra calculated at $12 \mathrm{~h}$ after trigger, assuming for all cases the same kinetic energy ( $\left.E=10^{53} \mathrm{erg}\right)$, the same $\epsilon_{B}=10^{-3}$ value, the same $\epsilon_{e}=10^{-1}$, and external density $\left(n=1 \mathrm{~cm}^{-3}\right.$ for the homogeneous ISM case and $\dot{M}=3 \times 10^{-6} M_{\odot} \mathrm{yr}^{-1}$ and $v=10^{3} \mathrm{~km} \mathrm{~s}^{-1}$ for the wind case). What changes is only the slope of the electron distribution $p$. As can be seen, we indeed

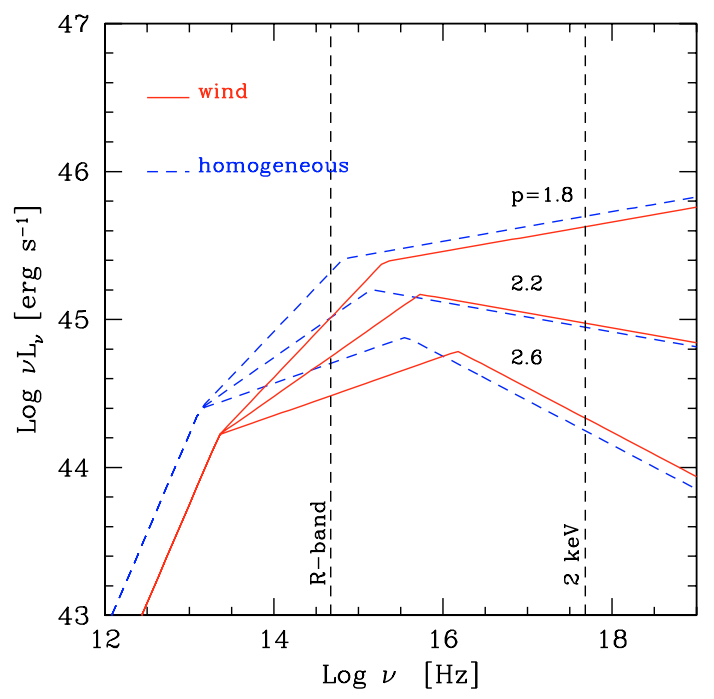

Fig. 15. Examples of spectra calculated using the prescriptions of Panaitescu \& Kumar (2000), at $12 \mathrm{~h}$ after trigger. Dashed lines correspond to a homogeneous ISM case (with density $n=1 \mathrm{~cm}^{-3}$ ); solid lines to a wind profile of the density (with $\dot{M}=3 \times 10^{-6} M_{\odot} \mathrm{yr}^{-1}$ and wind velocity $v=10^{3} \mathrm{~km} \mathrm{~s}^{-1}$. The models differ for the assumed values of $p$ (as labelled).

find in this case that the optical luminosities are distributed in a much narrower range than the X-ray luminosities. This is due to the fact that the cooling frequency changes with changing $p$ as a result of Compton losses being important, and decreasing for smaller $p$. Note also that this is true both for the homogeneous and the wind case.

We stress that this example is only illustrative, and does not pretend to give an exhaustive explanation of our results, since there can be other solutions in which more than one parameter is changing. Keeping this in mind, the observed clustering of the optical luminosities would then require that the kinetic (isotropically equivalent) energy is distributed in a narrow range, as are the equipartition parameters. Furthermore, $\epsilon_{B}$ (and/or the density $n$ ) should be small, and the Compton $Y$ parameter relatively large.

\subsection{Dark bursts}

Our results can help in understanding why a significant fraction of bursts with detected X-ray afterglows are not detected in the optical, even now in the SWIFT era, which allows very early optical observations both onboard through UVOT and on the ground through several robotic telescopes. Although our samples are still limited, it appears that there is a family of optically underluminous objects (dimmer by an order of magnitude than the average for the main family). These bursts are the obvious candidates for being missed in the optical, especially in the presence of absorption in the host galaxy (Nardini et al. in prep.).

We can wonder if these GRBs appear underluminous because of an underestimation of the intrinsic absorption. In this respect, we note that for GRB 011121 three different authors all estimate a null value of $A_{V}^{\text {host }}$, while they differ somewhat on the value of the Galactic extinction. To be conservative, we have taken the highest value of those. For GRB 021211, there are two estimates of $A_{V}^{\text {host }}$, so we used the conservative choice, taking the higher value. For GRB 980613 there is only one estimate of $A_{V}^{\text {host }}$, but a constraint on the maximum possible value of $A_{V}^{\text {host }}$ comes from its SED. From Fig. 10 it can be seen that the 
optical luminosity of this burst cannot be larger than a factor of ten from what is plotted, if we require a smooth joining of the extrapolated optical/X-ray spectra. This would barely bring this burst to the faint end of the luminous family. However, in this case the SED would appear anomalous, because the peak of the spectrum would be in the optical-near-UV (in contrast to the majority of the other bursts), and the optical spectral index resulting from such a large correction would be flatter than $\beta_{\mathrm{o}}=0.5$, again in contrast to the other bursts.

We do not know yet if these few underluminous bursts are the tip of the iceberg of much more numerous populations, and we do not know the corresponding spread in luminosities. We believe that SWIFT will clarify this point.

\section{Conclusions}

The main results of our study are:

- The optical luminosities of GRB afterglows, calculated at the same rest-frame time, show an unexpected tight clustering, with most $(21 / 24)$ of the optical luminosities spanning less than one order of magnitude around a mean value of $\log L_{v_{R}}=30.65$.

- A minority (3/24) of GRBs form a separate dimmer family, with an optical luminosity one order of magnitude less than for the more numerous family.

- These results were obtained considering all bursts with known redshift and optical extinction in the host galaxy, but the inclusion of the recently detected SWIFT bursts (of still unknown extinction in the host) is fully consistent with these findings, and reinforces them.

- The optical luminosity distribution appears narrower than the X-ray luminosity distribution of the same bursts, calculated at the same rest-frame time.

- The isotropic optical luminosities are not correlated with $E_{\gamma \text {,iso }}$.

- The X-ray isotropic luminosity correlates (even if not strongly) with the isotropic prompt emitted energy $E_{\gamma, \text { iso }}$, but this is simply due to the dependence of both quantities on the aperture angle of the jet. The collimation corrected prompt energy and X-ray luminosity are not correlated.

- The optical to X-ray SEDs of our bursts show that for most of the objects the entire observed emission is due to the same (synchrotron) process (after several hours to a day after trigger, rest-frame time). In a $v L_{v}$ plot, the peak frequency lies between the optical and the X-ray bands. The peak frequency can be identified with the cooling frequency.

- Our results are quite unexpected, and their interpretation is not obvious. One possibility points towards the importance of changing the slope of the electron energy distribution, while the other parameters are more constant.

Acknowledgements. We thank the Italian MIUR for funding (Cofin grant 2003020775_002).

\section{References}

Antonelli, L. A., Piro, L., Vietri, M., et al. 2000, ApJ, 545, L39 Atteia, J. L., Kawai, N., Vanderspek, R., et al. 2005, ApJ, 626, 292 Bartolini, G., Guarnieri, A., Piccioni, A., et al. 2003, GCN, 2030 Berger, E., \& Becker, G. 2005, GCN, 3520

Berger, E., Diercks, A., Frail, D. A., et al. 2001, ApJ, 556, 556 Berger, E., Kulkarni, S. R., Bloom, J. S., et al. 2002, ApJ, 581, 981 Berger, E., Kulkarni, S. R., \& Frail, D. A. 2003, ApJ, 590, 379 Berger, E., Kulkarni, S. R., Fox, D. B., et al. 2005a, ApJ, 634, 501 Berger, E., Cenko, S. B., Steidel, S. B., Reddy, N., \& Fox, D. B. 2005b, GCN, 3368 Bersier, D., McLeod, B., Garnavich, P. M., et al. 2003a, ApJ, 583, 63
Bersier, D., Stanek, K. Z., Winn, L. N., et al. 2003b, ApJ, 584, L43 (data table in [arXiv: astro-ph/0211130])

Beuermann, K., Hessman, F. V., Reinsch, K., et al. 1999, A\&A, 352, L26

Bhargavi, S. G., \& Cowslk 2000, ApJ, 545, L77

Bloom, J. S., Frail, D. A., Kulkarni, S. R., et al. 1998, ApJ, 508, 21

Bloom, J. S., Diercks, A., Djorgovski, S. G., Kaplan, D., \& Kulkarni, S. R. 2000, GCN, 661 Bloom, J. S., Frail, D. A., \& Kulkarni, S. R. 2003, ApJ, 594, 674

Bloom, J. S., van Dokkum, P. G., Bailyn, C. D., et al. 2004, AJ, 127, 252

Blustin, A. J., Band, D., Barthelmy, S., et al. 2005 [arXiv : astro-ph/0507515]

Boër, M., \& Gendre, B. 2000, A\&A, 361, L21

Brown, P., Retter, A., Schady, P., et al. 2005, GCN, 3549

Burenin, R., Denissenko, D., Pavlinsky, M., et al. 2003a, GCN, 2001

Burenin, R., Sunyaev, R., Pavlinsky, M., et al. 2003b, GCN, 2024

Burenin, R., Sunyaev, R., Pavlinsky, M., et al. 2003c, GCN, 2046

Burenin, R., Sunyaev, R., Pavlinsky, M., et al. 2003d, GCN, 2051

Burenin, R., Sunyaev, R., Pavlinsky, M., et al. 2003e, GCN, 2079

Burenin, R., Sunyaev, R., Denissenko, D., et al. 2003f, GCN, 2054

Burenin, R., Sunyaev, R., Pavlinsky, M., et al. 2003g, GCN, 2260

Butler, N. R., Marshal, H. L., Ricker, G. R., et al. 2003, ApJ, 597, 1010

Caldwell, N., Garnavich,, P., Holland, S., Matheson, T., \& Stanek, K. Z. 2003, GCN, 2053

Cameron, P. B., \& Frail, D. A. 2005, GCN, 4266

Cantiello, M., Dolci, M., Maiorano, E., et al. 2003, GCN, 2074

Castander, F. J., \& Lamb, D. Q. 1999, ApJ, 523, 602

Castro-Tirado, A. J., Zapatero-Osorio, M. R., Gorosabel, J., et al. 1999, ApJ, 511, L85

Fox, D. W., Cenko, S. B., \& Fox, D. W. 2005, GCN, 3834

Chapman, R., Tanvir, N., Rol, E., et al. 2005, GCN, 3375

Cobb, B. E., \& Bailyn, C. D. 2005a, GCN, 3104

Cobb, B. E., \& Bailyn, C. D. 2005b, GCN, 3110

Costa, E. 1999, A\&AS, 138, 425

Costa, E., Frintera, F., Heise, J., et al. 1997, Nature, 387, 783

Covino, S., Malesani, D., Tavecchio, F., et al. 2003, A\&A, 404, L5

Covino, S., Piranomonte, S., Fugazza, D., et al. 2005, GCN, 4046

D'Avanzo, P., Fugazza, D., Masetti, N., et al. 2005, GCN, 3171

D'Elia, V., Piranomonte, S., Fiore, F., et al. 2005, GCN, 4044

De Pasquale, M., Piro, L., Perna, R., et al. 2003, ApJ, 592, 1018

Djorgovski, S. G., Kulkarni, S. R., Goodrich, R., Frail, D., \& Bloom 1998, GCN, 137

Djorgovski, S. G., Kulkarni, S. R., Bloom, J. S., et al. 1999a, GCN, 189

Djorgovski, S. G., Kulkarni, S. R., Bloom, J. S., et al. 1999b, GCN, 289

Djorgovski, S. G., Mahabal, A., Price, P. A., et al. 2001, GCN, 1108

Diercks, A., Deutsch, E. W., Castander, F. J., et al. 1998, ApJ, 503, L105

Durig, D. T., \& Price, A. 2005, GCN, 4023

Durig, D. T., McLarty, N. P., \& Manning, J. R. 2005, GCN, 3950

Feng, M., Wang, L., \& Wheeler, J. C. 2000, GCN, 607

Fiore, F., Savaglio, S., Antonelli, L. A., et al. 2002, GCN, 1524

Fitzgerald, J. B., \& Orosz, J. A. 2003a, GCN, 2056

Fitzgerald, J. B., \& Orosz, J. A. 2003b, GCN, 2070

Foley, R. J., Chen, H. W., Bloom, J. S., \& Prochaska, J. X. 2005a, GCN, 3483

Foley, R. J., Chen, H. W., Bloom, J. S., \& Prochaska, J. X. 2005b, GCN, 3949

Fox, D. W., Price, P. A., Soderberg, A. M., et al. 2003, ApJ, 586, L5

Fox, D. W., \& Cenko, S. B. 2005, GCN, 3829

Frail, D. A., Kulkarni, S. R., Sari, R., et al. 2001, ApJ, 562, L55

Fynbo, J. P. U., Gorosabel, J., Dall, T. H., et al. 2001, A\&A, 373, 796

Fynbo, J. P. U., Hjorth, J., Jensen, B. L., et al. 2005a, GCN, 3136

Fynbo, J. P. U., Jensen, B. L., Jakobsson, P., et al. 2005b, GCN, 4040

Fynbo, J. P. U., Jensen, B. L., Hjorth, J., et al. 2005c, GCN, 3176

Fynbo, J. P. U., Jensen, B. L., Sollerman, J., et al. 2005d, GCN, 3874

Fugazza, D., Fiore, F., Patat, F., et al. 2005, GCN, 3948

Gal-Yam, A., Ofek, E. O., \& Lipkin, Y. 2002, GCN, 1335

Gal-Yam, A., Ofek, E. O., \& Lipkin, Y. 2003, GCN, 1999

Galama, T. J., Wijers, R. A. M. J., Bremer, M., et al. 1998, ApJ, 497, L

Galama, T. J., Briggs, M. S., Wijers, R. A. M. J., et al. 1999, Nature, 398, 394

Galama, T. J., Reichart, D., Brown, T. M., et al. 2003, ApJ, 587, 135

Garcia, M. R., Callanan, P. J., \& Moraru, D. 1998, ApJ, 500, 105

Garimella, K., Homewood, A., \& Hartmann, D. 2005, GCN, 4257

Garnavich, P. M., Jha, Stanek, K. Z., \& Garcia, M. 1999, GCN, 215

Garnavich, P. M., Jha, S., Pahre, M. A., et al. 2000, ApJ, 543, 61

Garnavich, P. M., Stanek, K. Z., Wyrzykowski, L., et al. 2003, ApJ, 582, 924

Gendre, B., \& Boër, M. 2005, A\&A, 430, 465 (GB05)

Giannini, T., Nisini, B., Antonelli, L. A., Fiore, F., \& Stella, L. 2002, GCN, 1678

Gladders, M., Holland, S., Garnavich, P. M., et al. 2001, GCN, 1209

Ghirlanda, G., Ghisellini, G., \& Lazzati, D. 2004, ApJ, 616, 331

Gorosabel, J. 2005, GCN, 3865

Greco, G., Bartolini, C., Guarnieri, A., et al. 2005, GCN, 3319

Greiner, J., Guenther, E., Klose, S., \& Schwarz, R. 2003a, GCN, 1886

Greiner, J., Klose, S., Salvato, M., et al. 2003b, ApJ, 599, 1223

Haislip, J., \& Reichart, D. 2005, GCN, 3719

Haislip, J., Reichart, D., Cypriano, E., et al. 2005, GCN, 3914

Halpern, J. P., \& Mirabal, N. 2005, GCN, 3907

Halpern, J. P., Uglesich, R., Mirabal, N., et al. 2000, ApJ, 543, 697

Halpern, J. P., Mirabal, N., Bureau, M., \& Fathi, K. 2003, GCN, 2021

Harrison, F. A., Bloom, J. S., Frail, D. A., et al. 1999, ApJ, 523, 121

Heise, J., DeLibero, C., Daniele, M. R., et al. 1999, IAUC, 7099

Hill, G., Prochaska, J. X., Fox, D., Schaefer, B., \& Reed, M. 2005, GCN, 4255

Hjorth, J. 2002, GCN, 1336

Hjorth, J., Thomsen, B., Nielsen, S. R., et al. 2002, ApJ, 576, 113

Hjorth, J., Moller, P., Gorosabel, J., et al. 2003, ApJ, 597, 699

Holland, S. T., Bersier, D., Bloom, J. S., et al. 2004, AJ, 128, 1955 
Holland, S. T., Björnsson, G., Hjorth, J., \& Thomsen, B. 2000, A\&A, 364, 467 Holland, S. T., Soszyński, I., Gladders, M. D., et al. 2002, AJ, 124, 639 Holland, S. T., Weidinger, M., Fynbo, J. P. U., et al. 2003, AJ, 125, 2291 Holman, M., Garnavich, P., \& Stanek, K Z. 2005, GCN, 3716

Huang, F. Y., Huang, K. Y., Urata, Y., Qiu, Y., \& Lou, Y. Q. 2005a, GCN, 4230 Huang, F. Y., Huang, K. Y., Urata, Y., Qiu, Y., \& Lou, Y. Q. 2005b, GCN, 4258 Ibrahimov, M. A., Asfandiyarov, I. M., Kahharov, B. B., et al. 2003a, GCN, 2077 Ibrahimov, M. A., Asfandiyarov, I. M., Kahharov, B. B., et al. 2003b, GCN, 2084 Ibrahimov, M. A., Asfandiyarov, I. M., Kahharov, B. B., et al. 2003c, GCN, 2098 Ibrahimov, M. A., Asfandiyarov, I. M., Kahharov, B. B., et al. 2003d, GCN, 2160 Ibrahimov, M. A., Asfandiyarov, I. M., Kahharov, B. B., et al. 2003e, GCN, 2191 Ibrahimov, M. A., Asfandiyarov, I. M., Kahharov, B. B., et al. 2003f, GCN, 2219 Ibrahimov, M. A., Asfandiyarov, I. M., Kahharov, B. B., et al. 2003g, GCN, 2288 Infante, L., Garnavich, P. M., Stanek, K. Z., \& Wyrzykowski, L. 2001, GCN, 1152 Israel, G. L., Marconi, G., Covino, S., et al. 1999, A\&A, 348, L5

Jakobsson, P., Hjorth, J., Fynbo, J. P. U., et al. 2004, A\&A, 427, 785 Jakobsson, P., Hjorth, J., Fynbo, J. P. U., et al. 2003, A\&A, 408, 941 Jelnek, M., de Ugarte Postigo, A., Castro-Tirado, A. J., et al. 2005, GCN, 4227

Jensen, B. L., Fynbo, J. U., Gorosabel, J., et al. 2001, A\&A, 370, 909

Jha, S., Pahre, M. A., Garnavich, P. M., et al. 2001a, ApJ, 554, 155 Jha, S., Matheson, T., Calkins, M., et al. 2001b, GCN, 974 Kawai, N., Yamada, T., Kosugi, G., Hattori, T., \& Aoki, K. 2005, GCN, 3937

Kahharov, B., Ibrahimov, M., Sharapov, D., et al. 2005, GCN, 3174

Kelson, D. D., \& Berger, E. 2005, GCN, 3101

Kelson, D. D., Franx, M., Magee, D., \& van Dokkum, P. G. 1999, IAUC, 7096

Khamitov, I., Aslan, Z., Golbasi, O., et al. 2003a, GCN, 2094

Khamitov, I., Bikmaev, I., Parmaksizoglu, M., et al. 2003b, GCN, 2198

Khamitov, I., Bikmaev, I., Galeev, A., et al., 2003c, GCN, 2299

Khamitov, I., Parmaksizoglu, M., Bikmaev, I., et al. 2003d, GCN, 2108

Khamitov, I., Parmaksizoglu, M., Uluc, K., et al. 2003e, GCN, 2119

Kindt, L., Andersen, H. H., \& Jakobsen, A. 2003, GCN, 2193

Kirschbrown, J., MacLeod, C., Reichart, D., et al. 2005, GCN, 3947

Klose, S., Stecklum, B., Masetti, N., et al. 2000, ApJ, 545, 271

Klose, S., Hoegner, C., \& Greiner, J. 2003a, GCN, 2000

Klose, S., Hoegner, C., \& Greiner, J. 2003b, GCN, 2029

Klose, S., Greiner, J., Rau, A., et al. 2004, AJ, 128, 1942

Klotz, A., Boér, M., \& Atteia, J. L. 2005a, GCN, 3403

Klotz, A., Boér, M., \& Atteia, J. L. 2005b, GCN, 3720

Kulkarni, S. R., Adelberger, K. L., Bloom, J. S., Kundic, T., \& Lubin, L. 1998, GCN, 29

Kumar, P., \& Piran, T. 2000, ApJ, 535, 152

Lachaume, R., \& Guyon 1999, IAUC, 7096

Lamb, D. Q., \& Reichart, D. E. 2000, ApJ, 536, 1

Laursen, L. T., \& Stanek, K. Z. 2003, ApJ, 597, L107

Lazzati, D., Covino, S., Ghisellini, G., et al. 2001, A\&A, 378, 996

Ledoux, C., Vreeswijk, P., Ellison, S., et al. 2005, GCN, 3860

Lee, B. C., Tucker, D. L., Vanden Berk, D. L., et al. 2001, ApJ, 561, 183

Lee, B. C., Lamb, D. Q., Tucker, D. L., \& Kent, S. 2003, GCN, 2096

Li, W., Chornock, R., Jha, S., \& Filippenko, A. V. 2003a, GCN, 2063

Li, W., Filippenko, A. V., Chornoch, R., \& Jha, S. 2003b, ApJ, 586, L12

Li, W., Filippenko, A. V., Chornoch, R., \& Jha, S. 2003c (The Astronomical Society Of The Pacific), 115,844

Lipkin, Y. M., Leibowitz, E. M., Ofek, E. O., Gal-Yam, A., \& Mandelson, H. 2003a, GCN 2049

Lipkin, Y. M., Leibowitz, E. M., Ofek, E. O., et al. 2003b, GCN, 2060

Lipkin, Y. M., Ofek, E. O., Gal-Yam, A., \& Mandelson, H. 2003c, GCN, 2034

Lipkin, Y. M., Ofek, E. O., Gal-Yam, A., Leibowitz, E. M., \& Mandelson, H. 2003d, GCN, 2045

Lipkin, Y. M., Ofek, E. O., \& Gal-Yam, A. 2004, ApJ, 606, 38

Lipunov, V., Krylov, A., Kornilov, V., et al. 2003, GCN, 2002

Lyuty, V., \& Metlov, V. 2003, GCN, 2113

McNaught, R., \& Price, P. A. 2005, GCN, 3163

Martini, P., Berlind, P., Stanek, K. Z., \& Garnavich, P. 2003, GCN, 2012

Masetti, N., Palazzi, E., Pian, E., et al. 1999, GCN, 233

Masetti, N., Palazzi, E., Pian, E., et al. 2001, A\&A, 374, 382

Masetti, N., Palazzi, E., Pian, E., et al. 2002, GCN, 1330

Masetti, N., Palazzi, E., Pian, E., et al. 2003, A\&A, 404, 465

Masetti, N., Palazzi, E., Pian, E., et al. 2005 [arXiv: astro-ph/0504592]

Masi, G., Mallia, F., Tagliaferri, U., et al. 2003, GCN, 2016

Matheson, T., Garnavich, P. M., Stanek, K. Z., et al. 2003, ApJ, 599, 394

Maury, A., Boér, M., \& Chaty, S. 1999, IAUC, 7099

Metzger, S. M., Djorgovski, S. G., Steidel, C.C., et al. 1997, IAUC, 6655

Milne, P. A., Williams, G. G., Park, H. S., \& Barthelmy, S. 2005a, GCN, 4218

Milne, P. A., Williams, G. G., Park, H. S., Barthelmy, S., \& Crist-Lair, J. 2005b, GCN, 4252

Mirabal, N., Paerels, F., \& Halpern, J. P. 2003, ApJ, 587, 128

Mirabal, N., Halpern, J. P. \& Tonnesen, S. 2005, GCN, 4215

Misra, K., Kamble, P., \& Pandey, S. B. 2005a, GCN, 3175

Misra, K., Kamble, P., Sahu, D. K., et al. 2005b, GCN, 4259

Mundell, C. G., Rol, E., Guidorzi, C., et al. 2005, GCN, 4250

Nousek, J. A., Kouveliotou, C., Grupe, C., et al. 2005, ApJ, submitted [arXiv: astro-ph/0508332]

Nysewander, M. C., Reichart, D. E., Park, H.-S., et al. 2005 [arXiv : astro-ph/0505474]
Odewahn, S. C., Bloom, J. S., \& Kulkarni, S. R. 1999, IAUC, 7094

Ofek, E., \& Leibowitz, E. M. 1999a, GCN, 210

Ofek, E., \& Leibowitz, E. M. 1999b, IAUC, 7096

Page, K. L., Beardmore, A. P., Goad, M. R., et al. 2005, GCN, 3837

Panaitescu, A., \& Kumar, P. 2000, ApJ, 543, 66

Panaitescu, A., \& Kumar, P. 2001, ApJ, 560, L49

Panaitescu, A., \& Kumar, P. 2002, ApJ, 571, 779

Pandey, S. B., Anupama, G. C., Sagar, R., et al. 2003a, A\&A, 408, L21

Pandey, S. B., Sahu, D. K., Resmi, L., et al. 2003b, Bull. Astron. Soc. India, 31

Pandey, S. B., Sagar, R., Anupama, G. C., et al. 2004, A\&A, 417, L919

Pavlenko, E., Rumyantsev, V., Antoniuk, O., Primak, N., \& Pozanenko, A. 2003a, GCN, 2067

Pavlenko, E., Rumyantsev, V., Antoniuk, O., Primak, N., \& Pozanenko, A. 2003b, GCN,

Pavlenko, E., Rumyantsev, V., Antoniuk, O., Primak, N., \& Pozanenko, A. 2003c, GCN, 2097

Perri, M., Capalbi, M., Giommi, P., et al. 2005, GCN, 3722

Pey, Y. C. 1992, ApJ, 395, 130

Piran, T., Kumar, P., Panaitescu, A., \& Piro, L. 2001, ApJ, 560, L167

Piro, L., Amati, L., Antonelli, L. A., et al. 1998, A\&A, 331, L41

Piro, L., Garmire, G., Garcia, M., et al. 2000, Science, 290, 955

Piro, L., Frail, D. A., Gorosabel, J., et al. 2002, ApJ, 577, 680

Pizzichini, G., Ferrero, P., Bartolini, C., et al. 2003, GCN, 2228

Price, A. 2003, GCN, 2058

Price, A., \& Mattei, J. 2003, GCN, 2071

Price, P. A., Berger, E., Kulkarni, S. R., et al. 2002a, ApJ, 573, L85 Price, P. A., Berger, E., Reichart, D. E., et al. 2002b, ApJ, 572, L51

Price, P. A., Kulkarni, S. R., Berger, E., et al. 2002c, ApJ, 571, L121

Price, P. A., Schmidt, B. P., \& Axelrod, T. S. 2002d, GCN, 1326

Price, P. A., Schmidt, B. P., \& Axelrod, T. S. 2002e, GCN, 1333

Price, P. A., Kulkarni, S. R., Schmidt, B. P., et al. 2003, ApJ, 584, L93

Prochaska, J. X., Bloom, J. S., Wright, J. T., et al. 2005, GCN, 3833

Quimby, R., Fox, D., Hoeflich, P., Roman, B., \& Wheeler, J. C. 2005, GCN, 4221 Reichart, D. E. 1999, ApJ, 521, L111

Rol, E., Tanvir, N., Levan, A., et al. 2005, GCN, 3372

Rumyantsev, V., Pavlenko, E., Antoniuk, O., \& Pozanenko, A. 2003a, GCN, 2028

Rumyantsev, V., Sergeeva, E., Doroshenko, V., et al. 2003b, GCN, 2146

Rykoff, E. S., \& Smith, D. A. 2003, GCN, 1995

Sagar, R., Pandey, A. K., Yadav, R. K. S., Nilakshi, \& Mohan, V. 1999, GCN, 227

Sato, R., Yatsu, Y., Suzuki, M., \& Kawai, N. 2003, GCN, 2080

Schlegel, D. J., Finkbeiner, D. P., \& Davis, M. 1998, ApJ, 500, 525

Sewmov, E. 2003, GCN, 2179

Sokolov, V. V., Kopylov, A. I., Zharikov, S. V., et al. 1998, A\&A, 334, 117

Sota, A., Castro-Tirado, A. J., Guziy, S., et al. 2005, GCN, 3705

Stanek, K. Z., Garnavich, P. M., Jha, S., et al. 2001, ApJ, 563, 592

Stanek, K. Z., Bersier, D., Calkins, M., Freedman, D. L., \& Spahr, T. 2003a, GCN, 2259

Stanek, K. Z., Martini, P., \& Garnavich, P. M. 2003b, GCN, 2041

Stanek, K. Z., Latham, D. W., \& Everett, D. M. 2003c, GCN, 2244

Stratta, G., Fiore, F., Antonelli, L. A., Piro, L., \& De Pasquale, M. 2004, ApJ, 608, 846

Suzuki, J., Sekiguchi, T., Miyasaka, S., et al. 2003, GCN, 2116

Starling, R. L. C., Vreeswijk, P. M., Ellison, S. L., et al. 2005, A\&A, 442, L21

Tagliaferri, G., Antonelli, L. A., Chincarini, G., et al. 2005 [arXiv: astro-ph/0509766]

Tiengo, A., Mereghetti, S., Ghisellini, G., Tavecchio, F., \& Ghirlanda, G. 2004, A\&A, 423, 861

Torii, K. 2003, GCN, 1986

Torii, K. 2005, GCN, 3943

Torii, K., Kato, T., Yamaoka, H., et al. 2003, ApJ, 597, L101

Urata, Y., Miyata, T., Nishiura, S., et al. 2003a, GCN, 2106

Urata, Y., Nishiura, S., Miyata, T., et al. 2003b, ApJ, 595, L24

Veillet, C. 1999a, GCN, 253

Veillet, C. 1999b, GCN, 260

Vietri, M. 1997, ApJ, 488, L105

Vreeswijck, P. M., Galama, T. J., Owens, A., et al. 1999a, A\&A, 523, 171

Vreeswijck, P. M., Galama, T. J., Stappers, B., et al. 1999b, GCN, 324

Vreeswijck, P. M., Rol, E., Hjorth, J., et al. 1999c, GCN, 496

Vreeswijck, P. M., Fruchter, A., Hjorth, J., \& Kouveliotou, C. 2003a, GCN, 1785

Vreeswijck, P. M., Wijers, R. A. M. J., \& Hjorth, J. 2003b, GCN, 1953

Vreeswijck, P. M., Ellison, S. L., Ledoux, C., et al. 2004, A\&A, 419, L927

Watson, D., Fynbo, J. P. U., Ledoux, C., et al. 2005 [arXiv: astrp-ph/0510368]

Weidinger, M., Fynbo, J. P. U., Hjorth, J., et al. 2003, GCN, 2215

Wijers, R. A. M. J., \& Galama, T. J. 1999, ApJ, 523, 177

Woźniak, P. R., Verstrand, W. T., Wren, J. A., et al. 2005, ApJ, 627, 13

Yadigaroglu, I. A., Halpern, J. P., Uglesich, R., \& Kemp, J. 1999, GCN, 242 Zeh, A., Klose, K., Laux, U., \& Greiner, J. 2003, GCN, 2048

Zharikov, S., \& Tovmassian, G. 2003, GCN, 2265

Zharikov, S., Benitez, E., Torrealba, J., \& Stepanian, J. 2003a, GCN, 2022

Zharikov, S., Benitez, E., Torrealba, J., \& Stepanian, J. 2003b, GCN, 2075

Zharikov, S., Tovmassian, G., \& Richer, M. 2003c, GCN, 2171

Zhu, J., \& Zhang, H. T. 1999a, GCN, 204

Zhu, J., \& Zhang, H. T. 1999b, IAUC, 7095 
M. Nardini et al.: Clustering of optical afterglow luminosities, Online Material $p 1$

\section{Online Material}




\section{Appendix A}

References for the data plotted in Figs. 1 and 4.

- GRB 970508: Garcia et al. (1998); Sokolov et al. (1998); Vietri et al. (1998).

- GRB 971214: Diercks et al. (1998).

- GRB 980613: Hjorth et al. (2002).

- GRB 980703: Bloom et al. (1998), Castro-Tirado et al. (1999), Vreeswijk et al. (1999).

- GRB 990123: Odewahn et al. (1999), (IAUC 7094); Zhu et al. (1999), (IAUC 7095); Zhu et al. (1999), (GCN 204); Lachaume et al. (1999), (IAUC 7096); Ofek et al. (1999), (GCN 210); Maury et al. (1999), (IAUC 7099); Garnavich et al. (1999), (GCN 215); Masetti et al. (1999), (GCN 233); Sagar et al. (1999), (GCN 227); Yadigaroglu et al. (1999), (GCN 242); Veillet (1999), (GCN 253); Veillet (1999), (GCN 260).

- GRB 990510: Harrison et al. (1999); Israel et al. (1999).

- GRB 991216: Garnavich et al. (2000); Halphern et al. (2000).

- GRB 000301c: Jensen et al. (2001); Bhargavi et al. (2000).

- GRB 000418: Berger et al. (2001).

- GRB 000911: Price et al. (2002); Lazzati et al. (2001); Masetti et al. (2005).

- GRB 000926: Fynbo et al. (2001); Price et al. (2001).

- GRB 010222: Galama et al. (2003).

- GRB 010921: Price et al. (2002), ApJ, 571, L121.

- GRB 011121: Greiner et al. (2003); Garnavich et al. (2003).

- GRB 011211: Jakobsson et al. (2003).

- GRB 020124: Hjorth et al. (2003); Berger et al. (2002).

- GRB 020405: Price et al. (2002), (GCN 1326); Price et al. (2002) (GCN 1333); Gal-Yam et al. (2002), (GCN 1335); Hjorth (2002), (GCN 1336).

- GRB 020813: Laursen \& Stanek (2003); Urata et al. (2003); Li et al. (2003).

- GRB 021004: Bersier et al. (2003); Pandey et al. (2003); Holland et al. (2003).

- GRB 021211: Holland et al. (2004); Pandey et al. (2003); Li et al. (2003b); Fox et al. (2003).

- GRB 030323: Vreeswijk et al. (2004).

- GRB 030226: KLose et al. (2004); Pandey et al. (2004).
- GRB 030329: Lipkin et al. (2004); Torii et al. (2003); Torii (2003), (GCN 1986); Rykoff (2003), (GCN 1995); Gal-Yam (2003), (GCN 1999), Klose et al. (2003), (GCN 2000); Burenin et al. (2003), (GCN 2001); Lipunov et al. (2003), (GCN 2002); Martini et al. (2003), (GCN 2012); Masi et al. (2003), (GCN 2016); Halpern et al. (2003), (GCN 2021); Zharikov et al. (2003), (GCN 2022); Burenin et al. (2003), (GCN 2024); Rumyantsev et al. (2003), (GCN 2028); Klose et al. (2003), (GCN 2029); Bartolini et al. (2003), (GCN 2030); Lipkin et al. (2003), (GCN 2034); Stanek et al. (2003), (GCN 2041); Lipkin et al. (2003), (GCN 2045); Burenin et al. (2003), (GCN 2046); Zeh et al. (2003), (GCN 2048); Lipkin et al. (2003), (GCN 2049); Burenin et al. (2003), (GCN 2051); Burenin et al. (2003), (GCN 2054); Fitzgerald et al. (2003), (GCN 2056); Price (2003), (GCN 2058); Lipkin et al. (2003), (GCN 2060); Li et al. (2003), (GCN 2063); Pavlenko et al. (2003), (GCN 2067); Fitzgerald et al. (2003), (GCN 2070); Price et al. (2003), (GCN 2071); Cantiello et al. (2003), (GCN 2074); Zharikov et al. (2003), (GCN 2075); Ibrahimov et al. (2003), (GCN 2077); Burenin et al. (2003), (GCN 2079); Sato et al. (2003), (GCN 2080); Pavlenko et al. (2003), (GCN 2083); Ibrahimov et al. (2003), (GCN 2084); Khamitov et al. (2003), (GCN 2094); Lee et al. (2003), (GCN 2096); Pavlenko et al. (2003), (GCN 2097); Ibrahimov et al. (2003), (GCN 2098); Urata et al. (2003), (GCN 2106); Khamitov et al. (2003), (GCN 2108); Lyuty et al. (2003), (GCN 2113); Suzuki et al. (2003), (GCN 2116); Khamitov et al. (2003), (GCN 2119); Rumyantsev et al. (2003), (GCN 2146); Ibrahimov et al. (2003), (GCN 2160); Zharikov et al. (2003), (GCN 2171); Semkov (2003), (GCN 2179); Ibrahimov et al. (2003), (GCN 2191); Kindt et al., (GCN 2193); Khamitov et al. (2003), (GCN 2198); Ibrahimov et al. (2003), (GCN 2219); Pizzichini at al. (2003), (GCN 2228); Stanek et al. (2003), (GCN 2244); Stanek et al. (2003), (GCN 2259); Burenin et al. (2003), (GCN 2260); Zharikov et al. (2003), (GCN 2265); Ibrahimov et al. (2003), (GCN 2288); Khamitov et al. (2003), (GCN 2299).

- GRB 030429; Jakobsson et al. (2004). 